\title{
Endogenous Rho-Kinase Signaling Maintains Synaptic Strength by Stabilizing the Size of the Readily Releasable Pool of Synaptic Vesicles
}

\author{
David González-Forero, ${ }^{1 *}$ Fernando Montero, ${ }^{1 *}$ Victoria García-Morales, ${ }^{1}$ Germán Domínguez, ${ }^{1}$ Laura Gómez-Pérez, ${ }^{1}$ \\ José Manuel García-Verdugo, ${ }^{2}$ and Bernardo Moreno-López ${ }^{1}$ \\ ${ }^{1}$ Grupo de Neurodegeneración y Neuroreparación (GRUNEDERE), Área de Fisiología, Facultad de Medicina, Universidad de Cádiz, Cádiz, Spain, and \\ ${ }^{2}$ Centro de Investigación Príncipe Felipe, CIBERNED, Universidad de Valencia, Valencia, Spain
}

\begin{abstract}
Rho-associated kinase (ROCK) regulates neural cell migration, proliferation and survival, dendritic spine morphology, and axon guidance and regeneration. There is, however, little information about whether ROCK modulates the electrical activity and information processing of neuronal circuits. At neonatal stage, ROCK $\alpha$ is expressed in hypoglossal motoneurons (HMNs) and in their afferent inputs, whereas ROCK $\beta$ is found in synaptic terminals on HMNs, but not in their somata. Inhibition of endogenous ROCK activity in neonatal rat brainstem slices failed to modulate intrinsic excitability of HMNs, but strongly attenuated the strength of their glutamatergic and GABAergic synaptic inputs. The mechanism acts presynaptically to reduce evoked neurotransmitter release. ROCK inhibition increased myosin light chain (MLC) phosphorylation, which is known to trigger actomyosin contraction, and reduced the number of synaptic vesicles docked to active zones in excitatory boutons. Functional and ultrastructural changes induced by ROCK inhibition were fully prevented/reverted by MLC kinase (MLCK) inhibition. Furthermore, ROCK inhibition drastically reduced the phosphorylated form of p21-associated kinase (PAK), which directly inhibits MLCK. We conclude that endogenous ROCK activity is necessary for the normal performance of motor output commands, because it maintains afferent synaptic strength, by stabilizing the size of the readily releasable pool of synaptic vesicles. The mechanism of action involves a tonic inhibition of MLCK, presumably through PAK phosphorylation. This mechanism might be present in adults since unilateral microinjection of ROCK or MLCK inhibitors into the hypoglossal nucleus reduced or increased, respectively, whole XIIth nerve activity.
\end{abstract}

\section{Introduction}

The serine/threonine Rho-associated kinase (ROCK), the major effector of the small GTP-binding protein RhoA, is pivotal for cell migration, proliferation, and survival. Through its regulatory role in actin cytoskeletal rearrangements, ROCK controls smooth-muscle contraction as well as cell migration, neurite outgrowth, and synapse retraction (Riento and Ridley, 2003; Mueller et al., 2005; Sunico et al., 2010; Moreno-López et al., 2011). Two isoforms of ROCK, I (or $\beta$ ) and II (or $\alpha$ ) have been described so far (Nakagawa et al., 1996). ROCK $\alpha$ is the main isoform found in the brain whereas $\operatorname{ROCK} \beta$ is preferentially expressed by non-

Received June 24, 2011; revised Oct. 4, 2011; accepted Nov. 9, 2011.

Author contributions: B.M.-L. and D.G.-F. designed research; D.G.-F., F.M., V.G.-M., G.D., L.G.-P., and J.M.G.-V. performed research; J.M.G.-V. contributed unpublished reagents/analytic tools; D.G.-F., F.M., V.G.-M., G.D., L.G.-P., and B.M.-L. analyzed data; B.M.-L. and D.G.-F. wrote the paper.

This work was supported by grants from Spain's Ministerio de Ciencia e Innovación (SAF2008-01415) and the Junta de Andalucía's Consejería de Innovación, Ciencia y Empresa (PAI2007-CTS-02606), both cofinanced by Fondo Europeo de Desarrollo Regional (B.M.-L.); Ministerio de Ciencia e Innovación (SAF2008-01274), Instituto de Salud Carlos III (RD06/0010/0022), and Conselleria de Sanitat-Centro de Investigación Príncipe Felipe to J.M.G.-V. F.M. received a Fellowship (AP2006-03925) from the Ministerio de Ciencia e Innovación, Spain. We thank Elaine Lilly, PhD (Writer's First Aid), for English language revision of this manuscript.

${ }^{*}$ D.G.-F. and F.M. contributed equally to this work.

Correspondence should be addressed to Dr. Bernardo Moreno-López, Área de Fisiología, Facultad de Medicina, Plaza Falla, 9, 11003 Cádiz, Spain. E-mail: bernardo.moreno@uca.es.

DOI:10.1523/JNEUROSCI.3215-11.2012

Copyright $\odot 2012$ the authors $\quad 0270-6474 / 12 / 320068-17 \$ 15.00 / 0$ neural tissue (Leung et al., 1995; Matsui et al., 1996; Nakagawa et al., 1996). Profuse distribution of ROCK $\alpha$ in neuron perikarya and dendrites of the frontal lobe, the hippocampus, and the cerebellum supports its contribution to essential brain functions (Hashimoto et al., 1999). ROCK participates in synaptic plasticity-underlined processes such as spatial learning, working memory, and fear memory consolidation (Dash et al., 2004; Huentelman et al., 2009; Ota et al., 2010). Both actin and ROCK, at the presynaptic and postsynaptic hippocampal synapse counterparts, are important for long-lasting potentiation (Wang et al., 2005). However, whether ROCK regulates neuronal physiology by modulating intrinsic membrane properties and/or afferent input drive to neurons remains unknown so far. In this way, ROCK regulates several ionic channels (Li et al., 2002; Piccoli et al., 2004; Staruschenko et al., 2004; Iftinca et al., 2007).

Phosphorylation of myosin light chain (p-MLC), one of the main substrates of ROCK, results in stimulation of actin-myosin interactions (Luo, 2002; Mueller et al., 2005), which are localized at presynaptic terminals (Drenckhahn and Kaiser, 1983). Actin filaments form an intricate cytoskeletal network that associates closely with vesicles and active zones (a.z.) (Hirokawa et al., 1989; Phillips et al., 2001). Actin, in turn, interacts with synapsin and captures vesicles (Sakaba and Neher, 2003), thereby preventing them from fusing to the plasma membrane (Llinás et al., 1985; Schiebler et al., 1986; Bähler and Greengard, 1987). 
The level of p-MLC is determined by the balanced actions of MLC kinase (MLCK) and MLC phosphatase (MLCP). In turn, ROCK can - directly and/or indirectly, by inhibition of MLCP phosphorylate MLC (Moreno-López et al., 2011). It is thus possible that ROCK modulates neurotransmitter release and electrical activity of neuronal circuits through its cytoskeletaldependent regulation of presynaptic vesicle pools. This hypothesis gains support from the finding that MLCK controls the size of the pool of fast releasing vesicles at the calyx of Held (Srinivasan et al., 2008).

The aim of this work was to scrutinize whether endogenous ROCK regulates motoneuron physiology by modulating intrinsic membrane properties and/or synaptic inputs to hypoglossal motoneurons (HMNs). The combined experimental analysis shows that presynaptic ROCK activity seems to maintain evoked neurotransmitter release from glutamatergic and GABAergic afferent inputs to HMNs. In view of our results, we propose a possible mechanism of action by which ROCK regulates synaptic strength. Finally, we provide evidence that ROCK activity is necessary for the normal performance of a motor output in the adult rat.

\section{Materials and Methods}

Wistar rats of either sex were obtained from an authorized supplier (Animal Supply Services, University of Cádiz, Spain), and were cared for and handled in accordance with the guidelines of the European Union Council (86/609/UE) and Spanish regulations (BOE 67/8509-12; BOE 1201/ 2005) on the use of laboratory animals. Experimental procedures were approved by the local Animal Care and Ethics Committee.

\section{Extraction of brainstem slices for in vitro experiments}

Rat pups (P6-P9) were anesthetized by hypothermia (placed on ice for $10-15 \mathrm{~min}$ ) and decapitated, and their brainstems were quickly removed (Sunico et al., 2010). Dissection was in ice-cold $\left(\sim 4^{\circ} \mathrm{C}\right)$ sucrose artificial CSF (S-aCSF) bubbled with $95 \% \mathrm{O}_{2}$ and $5 \% \mathrm{CO}_{2}$. S-aCSF composition was as follows (in mM): $26 \mathrm{NaHCO}_{3}, 10$ glucose, $3 \mathrm{KCl}, 1.25 \mathrm{NaH}_{2} \mathrm{PO}_{4}, 2$ $\mathrm{MgCl}_{2}$, and 218 sucrose. Transverse slices (300-400 $\mu \mathrm{m}$ thick) were obtained using a vibroslicer (NVSL; WPI). Slices were transferred to normal oxygenated aCSF (in ms: $26 \mathrm{NaHCO}_{3}, 10$ glucose, $3 \mathrm{KCl}, 1.25$ $\mathrm{NaH}_{2} \mathrm{PO}_{4}, 2 \mathrm{MgCl}_{2}, 130 \mathrm{NaCl}$, and $2 \mathrm{CaCl}_{2}$ ) and allowed to stabilize at $\sim 37^{\circ} \mathrm{C}$ for $30 \mathrm{~min}$. Next, slices were either transferred to a recording chamber for whole-cell patch-clamp recordings or incubated for $10 \mathrm{~min}$ $\left(\sim 22^{\circ} \mathrm{C}\right)$, with aCSF alone or supplemented with various drug treatments, before histological processing, hypoglossal nucleus (HN) microdissection for Western blotting experiments, or ROCK activity assay.

\section{Histological procedures}

For confocal microscopy. Animals were anesthetized with chloral hydrate, injected intraventricularly with heparin, and perfused transcardially first with PBS, followed by $4 \%$ paraformaldehyde (PFA) in $0.1 \mathrm{M}$ phosphate buffer $(\mathrm{PB}), \mathrm{pH} 7.4$, at $4^{\circ} \mathrm{C}$. The brains were removed and postfixed for $2 \mathrm{~h}$ in the same fixative solution. Brains were cryoprotected by overnight immersion in $30 \%$ sucrose in $0.1 \mathrm{M} \mathrm{PB}$ at $4^{\circ} \mathrm{C}$. Serial coronal sections ( $30 \mu \mathrm{m}$ thick) were obtained from brainstems using a microtome and stored at $-20^{\circ} \mathrm{C}$ in a cryoprotectant solution (glycerol/PBS, 1:1 v/v).

Immunohistochemistry was performed against synaptophysin (syn) and/or vesicular glutamate (VGLUT2) transporter as synaptic markers, ROCK $\alpha$ and ROCK $\beta$, and/or the nonphosphorylated form of neurofilament H (SMI32) as a motoneuron marker. Sections were rinsed in PBS and immersed in $2.5 \%(\mathrm{w} / \mathrm{v})$ bovine serum albumin, $0.25 \%(\mathrm{w} / \mathrm{v})$ sodium azide, and $0.1 \%(\mathrm{v} / \mathrm{v})$ Triton X-100 in PBS for $30 \mathrm{~min}$, followed by overnight incubation at $4^{\circ} \mathrm{C}$ with different combinations of up to three antisera. Polyclonal primary antibodies used in this study were anti-syn (1:200; Zymed Laboratories) developed in rabbit, anti-VGLUT2 (1:2000; Millipore Bioscience Research Reagents) developed in guinea pig, anti$\operatorname{ROCK} \alpha$ (1:50 or 1:100), anti-ROCK $\beta$ (1:50; Santa Cruz Biotechnology) developed in goat, and anti-SMI32 (1:8000; Covance) developed in mouse. Subsequently, the tissue was rinsed three times with PBS for 5 min each and incubated for $2 \mathrm{~h}$ at room temperature with the secondary antibodies, developed in donkey: anti-guinea pig, anti-goat, anti-mouse, or anti-rabbit IgGs labeled with the cyanine 2, 3, or 5 (Cy2, Cy3, Cy5) (1:200; Jackson ImmunoResearch Laboratories). Finally, sections were washed with PBS and mounted on slides with a solution containing propyl gallate $(0.1 \mathrm{~mm}$ in $\mathrm{PBS} /$ glycerol, $1: 9 \mathrm{v} / \mathrm{v})$. Omission of the primary antibodies resulted in no detectable staining.

Slides were analyzed using a Leica confocal microscope for fluorescence. All neurons were acquired in a $z$-plane where optimal antibody diffusion was reached. The pinhole opening was the same for all experimental conditions.

For electron microscopy. Brainstem slices ( $300 \mu \mathrm{m}$ thick) were fixed by $45 \mathrm{~min}$ immersion in $3.5 \%$ glutaraldehyde in $0.1 \mathrm{M} \mathrm{PB}, \mathrm{pH} 7.4$, at $36^{\circ} \mathrm{C}$. Slices were then left $30 \mathrm{~min}$ at room temperature before storing $20 \mathrm{~h}$ at $4^{\circ} \mathrm{C}$. Finally, the slices were rinsed six times with abundant $0.1 \mathrm{M} \mathrm{PB}$ and stored in $\mathrm{PB}$ with $0.05 \%$ sodium azide at $4^{\circ} \mathrm{C}$ until electron microscopy processing. Sections were postfixed with $2 \%$ osmium tetroxide in $0.1 \mathrm{M}$ PB for $90 \mathrm{~min}$. Subsequently, the slices were rinsed with distilled water, dehydrated in graded series of ethanol including 30\% (5 min), 50\% (5 $\mathrm{min}$ ), and $70 \%$ (10 $\mathrm{min}$ ), and stained for $2 \mathrm{~h} 30 \mathrm{~min}$ in $2 \%$ uranyl acetate in $70 \%$ ethanol at $4^{\circ} \mathrm{C}$. Afterward, sections were dehydrated again in graded series of ethanol including 70\% $(2 \times 5 \mathrm{~min}), 96 \%(2 \times 10 \mathrm{~min})$, and $100 \%(2 \times 10 \mathrm{~min})$, washed with propylene oxide $(2 \times 10 \mathrm{~min})$, embedded in Araldite resin, and left to polymerize for at least $72 \mathrm{~h}$ at $70^{\circ} \mathrm{C}$. Ultrathin sections $(70-80 \mathrm{~nm}$ thick) obtained with an ultramicrotome (LeicaEMUC6) were analyzed under a FEI Tecnai Spirit electron microscope at high magnification $(43,000 \times)$ attached to a digital camera (Morada Preview). Captured images were analyzed off-line using the ImageJ free software.

\section{Whole-cell patch-clamp recordings from HMNs in brainstem slices}

Whole-cell patch-clamp experiments were performed on HMNs from transverse brainstem slices of 6- to 9-d-old Wistar rats as previously described (González-Forero et al., 2007; Sunico et al., 2010). Recordings were performed at $31^{\circ} \mathrm{C}$ on slices superfused (rate $\sim 3-4 \mathrm{ml} / \mathrm{min}$ ) with aCSF solution equilibrated with $95 \% \mathrm{O}_{2}$ and $5 \% \mathrm{CO}_{2}$. HMNs were identified based on their location and characteristic size and shape and patched under visual guidance using IR-DIC (infrared-differential interference contrast) optics. Patch electrodes (1.5-3 $\mathrm{M} \Omega$ resistance) contained either a KGluconate-based internal solution (composition in mM: $17.5 \mathrm{KCl} ; 122.5$ KGluconate, $9 \mathrm{NaCl} ; 1 \mathrm{MgCl}_{2} ; 10$ HEPES; 0.2 EGTA; 3 Mg-ATP, 0.3 GTP-Tris with $\mathrm{pH}$ buffered to 7.2) or a CsCl-based internal solution (composition in mm: $120 \mathrm{CsCl} ; 4 \mathrm{NaCl} ; 4 \mathrm{MgCl}_{2} ; 1 \mathrm{Cl}_{2} \mathrm{Ca} ; 10$ HEPES; 0.2 EGTA; 3 Mg-ATP, 0.3 GTP-Tris). Current- and voltageclamp recordings were obtained and low-pass bessel-filtered at $10 \mathrm{kHz}$ with a MultiClamp 700B amplifier. Data were digitized at $20 \mathrm{kHz}$ with a Digidata 1332A analog-to-digital converter and acquired using pCLAMP 9.2 software (Molecular Devices). Only recordings with access resistance between 5 and $20 \mathrm{M} \Omega$ were considered acceptable for analysis. The access resistance was checked throughout the experiments, and recording was abandoned if it changed $>15 \%$. Series resistance was routinely compensated $65 \%$ to $75 \%$. Leak currents and liquid junction potentials were not corrected.

All drugs were prepared just before the experiment from stock solutions maintained at $-20^{\circ} \mathrm{C}$ and applied to the recording bath either alone, in combination, or in specific sequences by switching the perfusion line manually. In general, the protocol used for studying the effects of the drugs was as follows: all HMNs were initially perfused with normal aCSF to obtain baseline control data. After baseline preexposure recording, the slice was superfused for 10 min with aCSF containing a given drug before current or voltage responses were acquired again. In some experiments, a second drug was subsequently added to the perfusate and its effects tested $10 \mathrm{~min}$ later. Finally, a last round of acquisition was taken after a $10 \mathrm{~min}$ washout with drug-free aCSF. Data were stored on a computer disk and analyzed off-line by using MiniAnalysis (Synaptosoft) and Clampfit 9.2 (Molecular Devices) software. 
Current-clamp recordings. Current-clamp experiments were conducted to determine whether pharmacologic inhibition of endogenous ROCK activity affected intrinsic excitability and repetitive firing of HMNs. For current-clamp recordings, the patch pipettes were filled with the KGluconate-based pipette solution. The resting membrane potential $\left(V_{\mathrm{r}}\right)$, current threshold for firing $\left(I_{\mathrm{Th}}\right)$, input resistance $\left(R_{\mathrm{N}}\right)$, and relationship between average firing frequency and injected current $(f-I$ curves) were calculated for each cell under control preexposure conditions, during pharmacological treatment, and after washing out the drug. Depolarizing and hyperpolarizing current pulses were applied at $2 \mathrm{~s}$ intervals via the recording pipette. $I_{\mathrm{Th}}$ was determined as the lowest depolarizing current pulse $(5 \mathrm{~ms})$ required to elicit an action potential in $50 \%$ of cases. $R_{\mathrm{N}}$ was calculated from the current-voltage $(I-V)$ plots obtained by injecting a series of depolarizing and hyperpolarizing current pulses intracellularly $(0.5 \mathrm{~s} ;-0.2-0.2 \mathrm{nA})$. The resulting data points were then fitted with a least-squares regression line, and $R_{\mathrm{N}}$ was estimated as the slope of the lines. Finally, $f-I$ curves were constructed by applying intracellular injections of depolarizing current pulses ( $1 \mathrm{~s}$; steps of 0.02 $\mathrm{nA})$ and plotting the mean firing rate $(\mathrm{mFR})$ within each burst against current pulse amplitude. The curves were fitted with the three-parameter exponential equation $\mathrm{mFR}=a \cdot\left[1-e^{(-b \cdot \mathrm{I})}\right]+c$. The instantaneous slope of the curve was determined as the derivative of the exponential curve: slope $=a \cdot b \cdot e^{(-b \cdot \mathrm{I})}$. To compare the $f-I$ curves generated from the same cell under different experimental conditions, the slopes of the curves were computed at the midpoint of the current range tested.

Voltage-clamp recordings. Whole-cell voltage-clamp recordings were used to analyze the effects of pharmacologic inhibition of basal ROCK activity on synaptic strength at excitatory and inhibitory synapses on HMNs. To clearly determine these effects and avoid interference with other synaptic mechanisms, we focused our analysis on the AMPAergic component of the excitatory postsynaptic responses and on the $\mathrm{GABA}_{\mathrm{A}^{-}}$ receptor-mediated component of the inhibitory postsynaptic responses. To distinguish between presynaptic and postsynaptic effects of ROCK inhibition, we performed a combined electrophysiological analysis of (1) AMPAergic miniature EPSCs (mEPSCs ${ }_{\text {AMPA }}$ ), (2) spontaneous AMPA synaptic currents recorded under conditions of facilitated synaptic release $\left(\right.$ sEPSCs $\left._{\text {AMPA }}\right),(3)$ whole-cell AMPAergic current responses to glutamate pulses, (4) excitatory postsynaptic AMPA currents (eEPSCs $s_{\text {AMPA }}$ ), and inhibitory postsynaptic $\mathrm{GABA}_{\mathrm{A}}$ currents $\left(\mathrm{eIPSCs}_{\mathrm{GABA}}\right.$ ) evoked by electrical stimulation of afferent axons, (5) synaptic facilitation of eEPSCs ${ }_{\mathrm{AMPA}}$ following repetitive or paired-pulse stimulation, and (6) eEPSCs evoked by a minimal stimulation paradigm. Whole-cell AMPAergic responses were recorded at a holding potential of $-65 \mathrm{mV}$ with the KGluconate-based intracellular solution. GABA $_{\mathrm{A}}$ postsynaptic currents were recorded in cells voltage-clamped at $-70 \mathrm{mV}$ using the $\mathrm{CsCl}$-based electrode solution.

mEPSCs $_{\text {AMPA }}$ were pharmacologically isolated in the presence of $1 \mu \mathrm{M}$ tetrodotoxin (TTX), $1 \mu \mathrm{M}$ strychnine hydrochloride, $10 \mu \mathrm{M}$ bicuculline methochloride, $30 \mu \mathrm{M}$ D-tubocurarine, and $50 \mu \mathrm{M}$ (DL)-APV applied to the bath perfusion. All these drugs were purchased from Tocris Cookson.

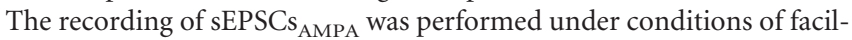
itated synaptic release without TTX in a modified extracellular solution containing high-Ca ${ }^{2+}(4 \mathrm{~mm})$, high- $\mathrm{K}^{+}(9 \mathrm{~mm})$, and the receptor antagonists indicated above. The AMPAergic nature of both types of events $\left(\mathrm{mEPSCs}_{\mathrm{AMPA}}\right.$ and sEPSCs $\mathrm{AMPA}_{\mathrm{AM}}$ ) was confirmed in some cells by blocking with NBQX (20 $\mu \mathrm{M}$; Tocris Cookson) at the end of the experiment. For each cell and treatment condition, we obtained segments of continuous recording (5-8 $\mathrm{min}$ ) of spontaneous activity. During analysis, the threshold level for detection of events was set at three times the root mean squares (RMS) noise ( $\sim 2-3 \mathrm{pA}$, detection threshold $\sim 6-9 \mathrm{pA})$. Spurious events and noise artifacts were manually rejected after visual inspection of the automatically screened events. For each cell, peak amplitudes and interevent intervals of single mEPSCs $_{\mathrm{AMPA}}$ and $\mathrm{sEPSCs}_{\mathrm{AMPA}}$ were measured across the different recording epochs and used to calculate mean values and generate cumulative probability plots.

To test sensitivities of HMNs to glutamate we placed, at 75-100 $\mu \mathrm{m}$ distance from the recorded cells, a second micropipette containing $1 \mathrm{~mm}$ glutamate (Tocris Cookson) coupled to a Picospritzer II (General Valve Corporation) pressure ejection system. The cells were voltage clamped at
$-65 \mathrm{mV}$ and tested for their response to $100 \mathrm{~ms}$ pulses of glutamate (20 psi). To avoid complications from other possible synaptic currents evoked by the glutamate pulse, aCSF was replaced with a modified extracellular solution containing nominally zero $\mathrm{Ca}^{2+}$. The AMPAergic component of the glutamate-evoked currents was pharmacologically isolated with bicuculline, strychnine, APV, and D-tubocurarine. For each cell we obtained one or two series of test pulses $(5-10$ at $0.05 \mathrm{~Hz})$ under each experimental condition. Traces were averaged and peak amplitude of response was measured for each treatment condition in each cell.

eEPSCs $_{\mathrm{AMPA}}$ and eIPSCs $\mathrm{GABA}_{\mathrm{GA}}$ were elicited by means of a concentric bipolar tungsten electrode placed in the ventrolateral reticular formation (VLRF) 0.5-1 mm from the border of the HN. Evoked AMPAergic or GABAergic responses were recorded in absence of TTX and pharmacologically isolated with blockers of glycine, nicotinic and NMDA receptors, plus either bicuculline or NBQX, respectively. Stimulation parameters $(<400 \mu \mathrm{A}, 50 \mu$ s pulse duration, delivered at $0.05 \mathrm{~Hz})$ were chosen to generate a sizable and reproducible postsynaptic response. Usually, the stimulus intensity was adjusted so that the postsynaptic response was $\sim 50 \%$ of maximal amplitude and then it was maintained constant throughout the recording period. A minimum of 10 responses was recorded under each pharmacological condition and averaged for analysis of eEPSCs ${ }_{\mathrm{AMPA}}$ or eIPSCs $\mathrm{GABA}_{\mathrm{Gm}}$ amplitudes. The effect of pharmacologic inhibition of basal ROCK activity on evoked glutamate release at VLRF inputs was also tested using a minimal-stimulation protocol. Pharmacologically isolated eEPSCs AMPA $_{\text {A }}$ were elicited by single pulses of minimum strength delivered at a frequency of $0.2 \mathrm{~Hz}$ via the VLRF stimulation electrode. "Minimal" stimulation was defined as a percentage of eEPSCs $_{\text {AMPA }}$ failures in the range between $30 \%$ and $40 \%$. The detection threshold was set at three times the RMS noise (threshold $9 \mathrm{pA}$ ). Evoked responses lower than the threshold level were counted as failures.

Facilitation of eEPSCs ${ }_{\text {AMPA }}$ was studied using conventional paired pulse and repetitive stimulation protocols. In these experiments, the AMPA- or $\mathrm{GABA}_{\mathrm{A}}$-receptor-mediated component of the responses was pharmacologically isolated as described above. For paired pulse facilitation, VLRF was stimulated with pairs of electrical stimuli with interpulse intervals ranging from $25-200 \mathrm{~ms}$ in $25 \mathrm{~ms}$ steps given with $30 \mathrm{~s}$ delay between pairs. At least 10 individual responses were averaged at each interval. Paired-pulse facilitation ratio (PPF ratio) was computed by dividing the peak amplitude of the second response by the peak amplitude of the first one. The repetitive stimulation protocol consisted of a train of 20 pulses at $40 \mathrm{~Hz}$ applied to the VLRF. For averaging, 5-10 trains were applied, with 60 s intervals between successive trains. The peak amplitude of the individual response throughout the train was determined from the average trace. Estimates of facilitation are expressed relative to the amplitude of first response in the train.

\section{Western blotting}

Tissue slices from neonatal rat pups (P6-P9) were obtained as described above and incubated for $10 \mathrm{~min}$ in carbogen-bubbled aCSF alone (control) or supplemented with drug treatments. Next, slices were immediately transferred to ice-cold $\left(\sim 4^{\circ} \mathrm{C}\right)$ aCSF supplemented with protease (1 mM phenylmethylsulfonyl fluoride, $10 \mathrm{mg} / \mathrm{ml}$ leupeptin, $10 \mathrm{mg} / \mathrm{ml}$ pepstatin $\mathrm{A}, 10 \mathrm{mg} / \mathrm{ml}$ aprotinin) and phosphatase inhibitors, where microdissection of HNs was performed. Using a $1 \mathrm{ml}$ syringe, microdissected nuclei were homogenized in lysis buffer [50 mM Tris/ $\mathrm{HCl}, \mathrm{pH} 7.4,1 \%$ $(\mathrm{v} / \mathrm{v})$ Triton X-100, 0.5\% (w/v) sodium deoxycholate] supplemented with protease and phosphatase inhibitors. Equal amounts of protein were processed for SDS-PAGE and immunoblotting, using a specific antibody against p-MLC and MLC developed in goat and rabbit, respectively (1:200; Santa Cruz Biotechnology), or phospho-p21 activated kinase $1-3\left(\mathrm{p}-\mathrm{PAK}_{1-3}\right)$ developed in rabbit (1:1000; Cell Signaling Technology). Membranes were also probed with anti- $\alpha_{1}$-tubulin antibody (1:250,000; Sigma-Aldrich) as control for the total amount of protein contained in each well. Analysis was performed using the ImageJ $1.36 \mathrm{~b}$ software from the National Institutes of Health.

\section{Electroneurographic recordings in adult animals}

Adult animals were prepared for electroneurographic recordings of both XIIth nerves as described previously (González-Forero et al., 2004; Mon- 
tero et al., 2008). Briefly, rats were anesthetized (1.5-3\% isoflurane mixed with $\left.100 \% \mathrm{O}_{2}\right)$ and additionally injected with atropine $(0.2 \mathrm{mg} / \mathrm{kg}$, i.m.). Teflon-isolated silver bipolar electrodes were fixed around both XIIth nerves. Electrodes were electrically isolated from neighboring tissue with Vaseline jelly and Parafilm. Rectal temperature was continuously monitored and maintained at $37 \pm 1^{\circ} \mathrm{C}$.

Spontaneous activity from both XIIth nerves was recorded in monopolar mode, AC coupled, amplified, and filtered $(10 \mathrm{~Hz}$ to $10 \mathrm{kHz})$. The electroneurographic signals were integrated $(\tau=20 \mathrm{~ms})$, and the area of each burst was determined using a parabola automatically fitted to the integrated burst activity provided by the Chart software (ADInstruments). The ratio between the treated and contralateral (control) sides was measured before and after the injection of ROCK inhibitors into the right $\mathrm{HN}$.

Glass micropipettes with tips broken to $\sim 25 \mu \mathrm{m}$ were used to perform unilateral microinjections of the vehicle (PBS) or ROCK inhibitors. Solution was injected $200 \mu \mathrm{m}$ lateral to the midline and $1.2 \mathrm{~mm}$ below the dorsal surface of the medulla at the obex level. Intranuclear microinjections were performed over $5 \mathrm{~min}$ intervals using an oil-filled system (Montero et al., 2010).

\section{ROCK activity assay}

Brainstem slices were obtained and incubated as indicated above. The HNs were microdissected and immediately immersed in $20 \mathrm{~mm}$ Tris, $\mathrm{pH}$ $8,250 \mathrm{~mm}$ sucrose $(70 \mu \mathrm{l})$. The tissue was then homogenized using an insulin syringe, and subsequently centrifuged at $1500 \times g$ for 2 min to remove the nuclei. Supernatant was used to measure ROCK activity using the 96-well ROCK Activity Assay Kit (Cell Biolabs) according to the manufacturer's instructions.

\section{Drugs and treatments}

Either alone or in different combinations, the following drugs were added to the incubation/recording bath: the specific inhibitors of ROCK trans-4-[(1R)-1-aminoethyl]-N-4-pyridinylcyclohexanecar boxamide dihydrochloride (Y-27632) and $(S)-(+)-2-m e t h y l-1-[(4-m e t h y l-5-$ isoquinolinyl)sulfonyl]-hexahydro- $H$-1,4-diazepine dihydrochloride (H-1152) (both at $20 \mu \mathrm{M}$; Tocris Bioscience), and the MLCK inhibitor 1-(5-Iodonaphthalene-1-sulfonyl)-1 H-hexahydro-1,4-diazepine hydrochloride (ML-7) (10 $\mu \mathrm{M}$; Sigma). All studies were performed at least 5-10 min after addition of drugs to the bath solution. In the experiments in adult animals, a solution containing Y-27632 (5 mM), H-1152 (2 mM), or ML-7 (2.5 $\mathrm{mm}$ ) was injected into the HN.

\section{Statistics and data analysis}

Data are expressed as the mean \pm SEM. For construction of cumulative frequency histograms of spontaneous AMPAergic current amplitudes, we collected a significantly high number of events from each neuron (between 4993 and 5397 for each pharmacological treatment). The number of analyzed boutons, a.z., or HMNs per experimental condition is indicated in figure legends or in the Results. Data were obtained from at least three animals per experimental condition. In Western blotting experiments, each individual assay was performed by using tissue samples collected from six neonatal animals per experimental condition. Quantitative data from Western blot represent three independent experiments. Applied statistical tests per experimental condition are indicated in figure legends or in results. In all cases, the minimum significance level was set at $p<0.05$.

\section{Results}

ROCK is expressed in the HN of neonatal animals and exhibits basal activity in in vitro slices

To explore a possible role of endogenous ROCK in shaping the normal motor output of the HN, we first studied the expression pattern of ROCK $\alpha$ and ROCK $\beta$ in the HN from neonatal (P7) rats. ROCK $\alpha$ immunohistochemistry labeled a large number of neuronal somata in the nucleus (Fig. $1 A$ ). Double immunolabeling with antibodies against $\operatorname{ROCK} \alpha$ and the motoneuronal marker SMI32 further confirmed that this isoform was expressed in HMN cell bodies (Fig. 1A, inset). Inspection of ROCK $\alpha$ im- munostaining at higher magnification also revealed the presence of ROCK $\alpha$-immunoreactive (ir) puncta-like structures in the $\mathrm{HN}$, which in some cases colocalized with the synaptic marker syn (Fig. $1 B$ ). On the other hand, ROCK $\beta$ labeling consisted of punctate staining densely distributed throughout the nucleus (Fig. 1C), in some examples delineating neuron-like profiles, and around SMI32-identified HMNs (Fig. $1 C$, inset). ROCK $\beta$-ir punctate also partially colocalized with syn (Fig. $1 D$ ). Our quantitative analysis revealed that $7.4 \pm 0.9 \%(n=65$ HMNs $)$ of syn-ir puncta apposed to the perikarya of HMNs showed ROCK $\beta$-ir, whereas only $0.5 \pm 0.2 \%(n=55 \mathrm{HMNs})$ coexpressed $\operatorname{ROCK} \alpha$. These data suggest that $\operatorname{ROCK} \alpha$ is the main isoform expressed in HMNs, whereas ROCK $\beta$ is the predominant isoform expressed in synaptic structures.

ROCK activity was measured in microdissected HN from rat pups (P7) using a commercial assay kit. Kinase activity was well fitted by a simple exponential equation $(r>0.9 ; p<$ 0.0001 ) relative to the protein amount tested (Fig. $1 E$ ), reaching a maximal value $(100.0 \pm 3.3 \%)$ at $0.75 \mu \mathrm{g}$. Preincubation of tissue samples with the specific ROCK inhibitor H-1152 for 10 min reduced ROCK activity in a dose-dependent manner, with $\sim 62 \%$ inhibition at $20 \mu \mathrm{M}$ and nearly full ROCK inhibition at $100 \mu \mathrm{M}$ (Fig. $1 E$; Mann-Whitney $U$ test, $p<0.05$ ). Therefore, these data indicate that the two ROCK isoforms are expressed in the $\mathrm{HN}$ at neonatal ages and that at least one of them retains endogenous kinase activity in our in vitro brainstem slice model.

Effects of ROCK inhibition on intrinsic excitability of HMNs The presence of ROCK $\alpha$ immunolabeling in HMNs raises the possibility that this kinase may directly modulate their intrinsic membrane properties. To test this, we performed whole-cell patch-clamp recordings of HMNs from sliced neonatal (P6-P9) rat brainstems and examined the effects of pharmacologic inhibition of ROCK on their intrinsic excitability. Current-clamp responses were compared before and after $10 \mathrm{~min}$ treatment with H-1152 $(20 \mu \mathrm{M})$. We found that inhibition of ROCK signaling had no apparent effect ( $p \geq 0.4$, paired $t$ test; $n=9 \mathrm{HMNs}$ ) on $V_{\mathrm{r}}$ $(-65.0 \pm 1.9 \mathrm{mV}$ before vs $-64.9 \pm 1.8 \mathrm{mV}$ after $\mathrm{H}-1152), R_{\mathrm{N}}$ $(80.2 \pm 12.8 \mathrm{M} \Omega$ before vs $78.9 \pm 10.8 \mathrm{M} \Omega$ after $\mathrm{H}-1152)$, or $I_{\mathrm{Th}}$ $(1050 \pm 103.2$ pA before vs $982.6 \pm 82.3$ pA after H-1152) (Fig. $1 F, G)$. To examine whether $\mathrm{H}-1152$ altered repetitive discharge of HMNs, we recorded the voltage responses to a series of suprathreshold depolarizing current steps (Fig. $1 H$ ). The resulting $f-I$ relationships were best fitted with three-parameter exponential rise to maximum functions $(r>0.9 ; p<0.001)$. Comparison of $f-I$ curves shows that the ROCK inhibitor did not modify repetitive firing properties of HMNs (Fig. 1I). The average instantaneous slope calculated at the midpoint of the curve was similar ( $p=0.79$, paired $t$ test, $n=8 \mathrm{HMNs}$ ) before and after drug perfusion $(53.1 \pm 5.4 \mathrm{~Hz} / \mathrm{nA}$ vs $52.8 \pm 5.7 \mathrm{~Hz} / \mathrm{nA}$, respectively). Therefore, these data do not support the idea that endogenous activity of ROCK modulates intrinsic excitability of neonatal HMNs, or at least not in our in vitro experimental model.

\section{Inhibition of endogenous ROCK signaling depresses excitatory synaptic transmission in $\mathrm{HMNs}$}

Given that our immunohistochemical studies revealed expression of ROCK protein in both HMNs and puncta-like structures resembling presynaptic boutons, we next examined the potential role of basal ROCK signaling in synaptic transmission. As we have recently reported (Sunico et al., 2010), electrical stimulation of 

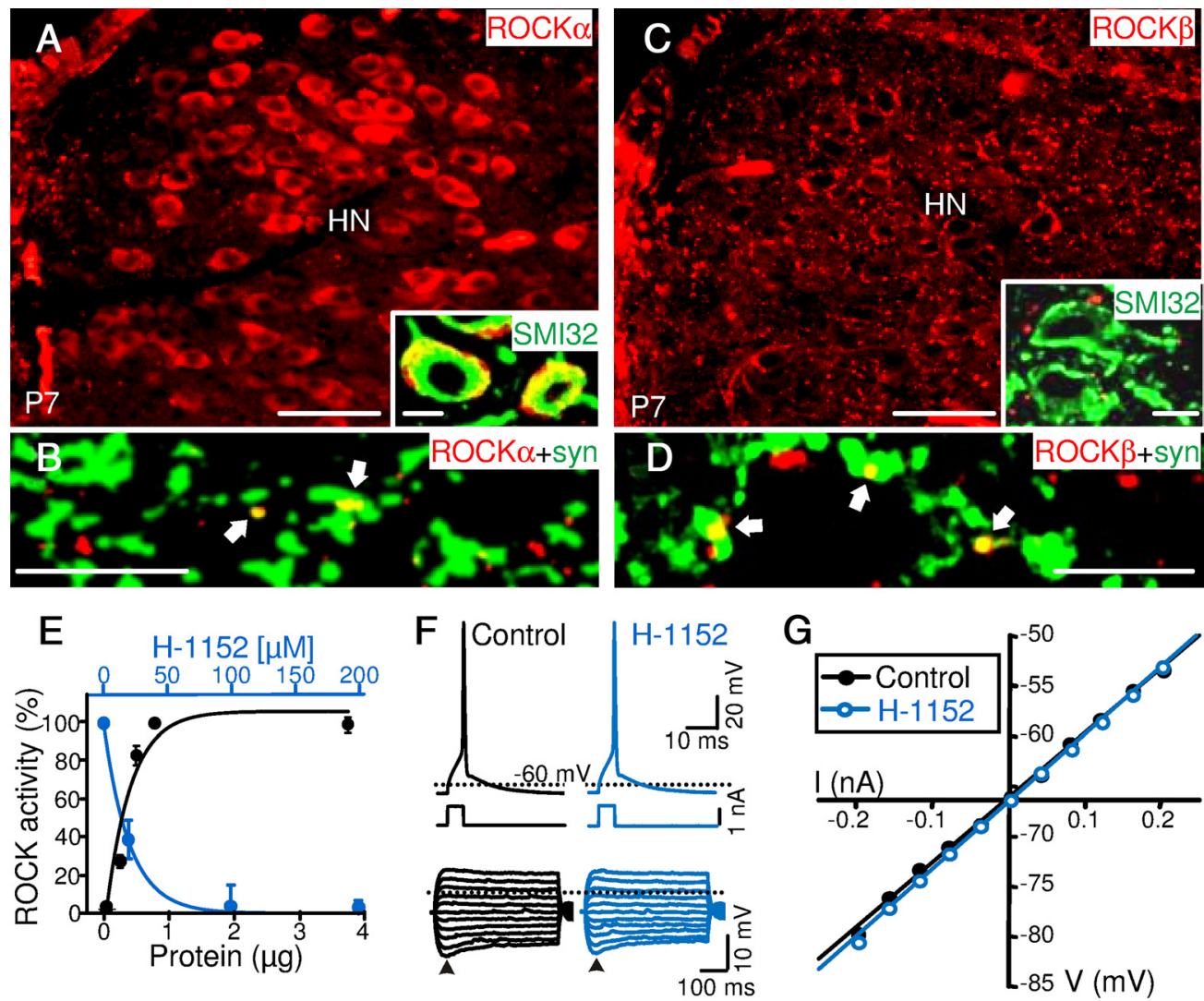
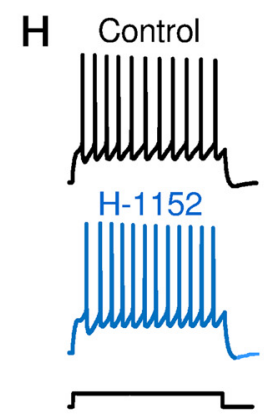
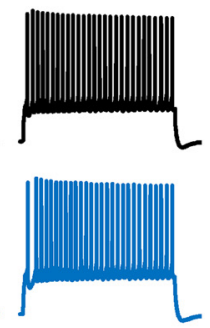

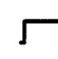
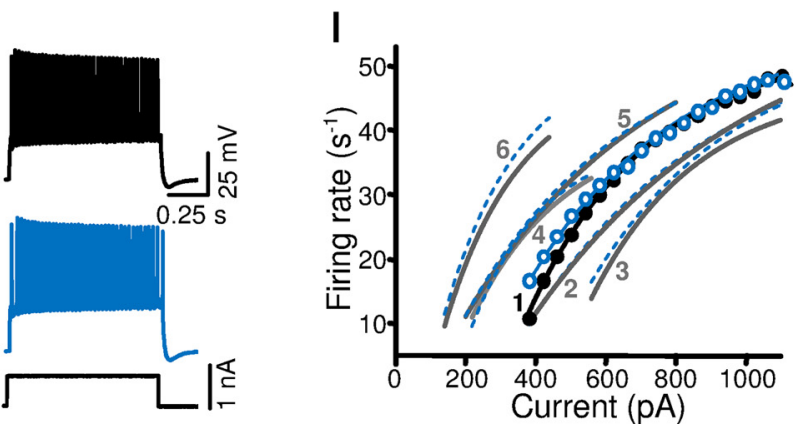

Figure 1. Inhibition of ROCK signaling had no effects on intrinsic excitability of HMNs. A-D, Low-magnification epifluorescence images showing the overall expression pattern of the two ROCK isoforms, ROCK $\alpha(\boldsymbol{A})$ and ROCK $\beta(\boldsymbol{C})$, in the HN from neonatal (P7) rats. Double-labeling confocal immunofluorescence for the synaptic marker synaptophysin (syn; green) and ROCK $\alpha(\boldsymbol{B})$ or ROCK $\beta(D)$ isoforms. Arrows indicate examples of colocalization of syn and ROCK $\alpha$ or $\beta$. Insets, Double-labeling confocal immunofluorescence for the motoneuron marker SMI32 (green) and ROCK $\alpha(\boldsymbol{A})$ or ROCK $\beta(C)$ isoforms (red). Scale bars: $A, C, 100 \mu \mathrm{m} ; \boldsymbol{B}, \boldsymbol{D}$, and insets, $10 \mu \mathrm{m}$. $\boldsymbol{E}$, ROCK activity relative to total protein content (black) and concentration of the ROCK inhibitor H-1152 (blue) determined in HN homogenates from neonatal rat pups. Each point represents the average from three independent assays. $\boldsymbol{F}$, Top traces, comparison of the voltage responses to identical depolarizing current pulses of threshold intensity obtained in an HMN before (control) and 10 min after adding $20 \mu \mathrm{m}$ the ROCK inhibitor to the bath (H-1152). Bottom traces, Subthreshold voltage responses of the same HMN to negative and positive current pulses ( $0.5 \mathrm{~s}$ duration, $0.04 \mathrm{nA}$ increments) under the specified recording conditions. Dotted lines indicate $-60 \mathrm{mV}$ and arrowheads the time point used to measure the peak voltage response. $G, V-I$ relationships for the traces shown in $F$. $R_{N}$ was determined by the slope of the regression line through the $V-I$ plot. The calculated $R_{N}$ for the $H M N$ was $64.5 \mathrm{M} \Omega$ in control conditions and $66.9 \mathrm{M} \Omega$ after drug perfusion. $\boldsymbol{H}$, Repetitive firing of an HMN for three current steps of increasing amplitude before (control) and after superfusion with $\mathrm{H}-1152$. I, Plot of mean $\mathrm{f}-$ / relationship for the HMN shown in $\boldsymbol{H}$ in control conditions (dots) and in the presence of H-1152 (circles). A collection of exponential regression lines for five additional HMNs (denoted by numbers) obtained before (solid gray lines) and after $\mathrm{H}-1152$ treatment (dashed blue lines) are displayed in the graph as well.

the VLRF evokes monosynaptic EPSPs in HMNs in in vitro preparations (Fig. $2 \mathrm{~A}$ ). In the voltage-clamp mode, application of the same type of stimulus consistently evoked large, inwardly directed, postsynaptic currents in HMNs held at $-65 \mathrm{mV}$ using KGluconate-filled electrodes. This response was almost fully suppressed with antagonists for AMPA (NBQX) and NMDA (APV) receptors (Fig. $2 A$ ). The small residual current component persisting in the presence of these blockers was partially abolished with the specific $\mathrm{GABA}_{\mathrm{A}}$ - and glycine-receptor antagonists bicuculline and strychnine, respectively (Fig. $2 A$ ).
To test whether endogenous ROCK signaling modulates excitatory synaptic transmission from these afferent inputs, we analyzed the effect of specific ROCK inhibitors on the electrically evoked EPSCs in HMNs. In vivo, most HMNs exhibit a repetitive rhythmic firing consisting of inspiratory-related bursting discharges driven by excitatory brainstem afferences (Peever et al., 2002; González-Forero et al., 2004). The inspiratory synaptic drive to HMNs is mediated by the excitatory amino acid glutamate mainly acting on AMPA receptors (AMPARs) (Rekling and Feldman, 1998). Hence, we focused our functional analysis on 

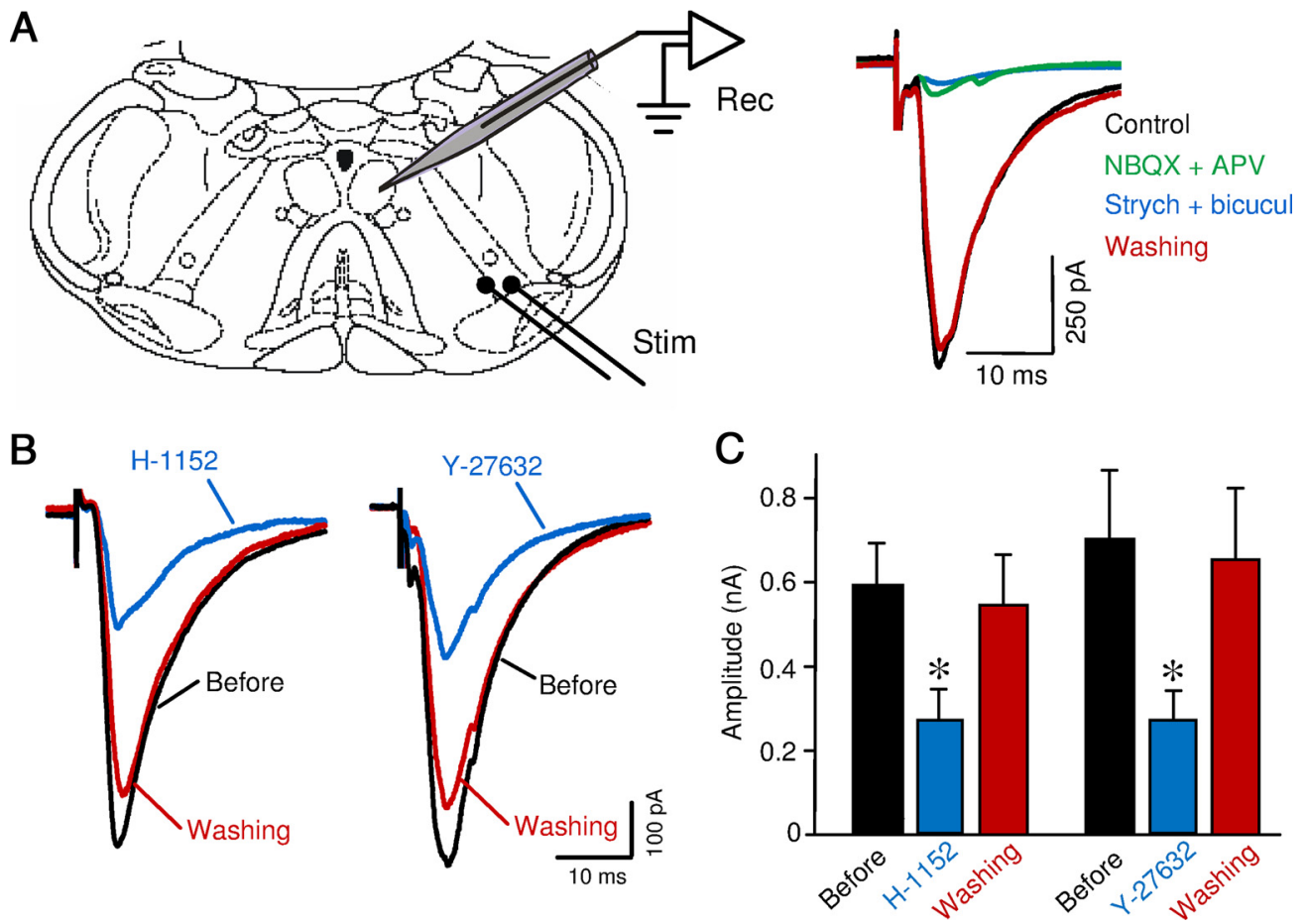

Figure 2. Pharmacologic inhibition of basal ROCKstrongly reduces the amplitude of the AMPAergic component of the EPSCs in HMNs. A, Left, schematic diagram of the in vitro experimental model used to analyze the effects of pharmacologic inhibition of ROCK activity on synaptic transmission to HMNs. Whole-cell patch-clamp recordings (Rec) were obtained from somata of large HN neurons in neonatal brainstem slices. EPSCs were evoked by electrical stimulation (Stim) of the ventrolateral reticular formation (VLRF). Right, Pharmacological dissection of postsynaptic currents evoked in HMNs by electrical stimulation of the VLRF. Cells were voltage-clamped at $-65 \mathrm{mV}$. Addition of glutamate receptor blockers ( $20 \mu \mathrm{m} \mathrm{NBQX}+50 \mu \mathrm{m}$ APV) reduced by $>90 \%$ the full current peak amplitude. The remaining residual current was partially abolished with $\mathrm{GABA}_{\mathrm{A}}$ - and glycine-receptor antagonists (10 $\mu \mathrm{m}$ bicuculline $+1 \mu \mathrm{m}$ strychnine). $\boldsymbol{B}$, Representative examples of pharmacologically isolated eEPSC $S_{A M P A}$ from HMNs recorded before (black), following a 10 min exposure to $\mathrm{H}-1152$ or Y-27632 (blue), and after 10 min of wash with drug-free solution (red). C, Summary

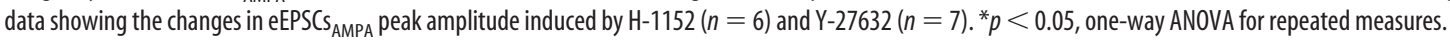

the AMPAergic component of the EPSCs evoked by VLRF stimulation (eEPSCs AMPA $_{\text {AM }}$ ) and tested its sensitivity to H-1152 or Y-27632. The results show that the amplitude of the eEPSCs $s_{\text {AMPA }}$ was strongly attenuated (by $>50 \%$ ) after 10 min continuous infusion with either of these drugs (from $-591.8 \pm 103.6$ to $-214.9 \pm 77.6 \mathrm{pA}$ for $\mathrm{H}-1152$, and from $-699.3 \pm 159.7$ to $-267.6 \pm 74.0 \mathrm{pA}$ for Y-27632) (Fig. 2B,C). This effect was reversed upon washing with drug-free aCSF. In summary, these results indicate that endogenous activity of ROCK is required to maintain the strength of AMPAR-mediated synaptic transmission in the HN.

\section{Endogenous ROCK signaling operates presynaptically to facilitate glutamate release}

To dissect the synaptic site of action for ROCK inhibitors, we performed a combined electrophysiological analysis. Miniature quantal events represent the postsynaptic responses to transmitter release from single vesicles, and their average amplitude is usually taken as a measure of the sensitivity and/or number of postsynaptic receptors (Turrigiano et al., 1998; Carroll et al., 1999; González-Forero and Alvarez, 2005). In the first set of experiments, we examined the consequences of inhibiting endogenous ROCK activity with $\mathrm{H}-1152$ on pharmacologically isolated mEPSCs $_{\text {AMPA }}$. The mEPSCs ${ }_{\text {AMPA }}$ were totally blocked by bath coapplication of NBQX, confirming that they were AMPARmediated events (data not shown). Our data show that application of H-1152 had no significant effect on the EEPSC $_{\text {AMPA }}$ amplitude (Fig. $3 A$ ). Thus, the mean amplitude of mEPSCs ${ }_{\text {AMPA }}$ recorded after 10 min exposure to this drug was $23.3 \pm 0.9 \mathrm{pA}$, which was comparable to the average amplitude of $\mathrm{mEPSC} \mathrm{s}_{\mathrm{AMPA}}$ measured under preexposure control conditions $(23.8 \pm 1.1 \mathrm{pA}$; $p=0.74$, paired $t$ test; $n=6 \mathrm{HMNs}$ ). This was also supported by the drug's lack of effect on the cumulative probability functions of $\mathrm{mEPSC}_{\mathrm{AMPA}}$ amplitudes (Fig. $3 B$; $p=0.25$; KolmogorovSmirnov test). Likewise, $\mathrm{H}-1152$ did not have any marked effect on the average frequency of mEPSCs $_{\mathrm{AMPA}}(6.6 \pm 1.4 \mathrm{~Hz}$ before vs $6.4 \pm 2.0 \mathrm{~Hz}$ after $\mathrm{H}-1152$ ).

To further investigate whether the facilitatory effect of basal ROCK signaling on AMPAergic synaptic transmission occurs through a postsynaptic mechanism, HMNs were tested for their sensitivity to exogenously applied glutamate pulses before and after H-1152 application. Exogenous glutamate pulses consistently evoked large inward AMPAergic currents in all HMNs tested (Fig. 3C), with a mean value of $-4.73 \pm 0.49 \mathrm{nA}(n=7$ HMNs). Bath perfusion of $\mathrm{H}-1152$ had no significant effect ( $p=$ 0.78 , paired $t$ test) on the mean peak amplitude of the glutamateevoked currents $(-4.67 \pm 0.44 \mathrm{nA})$ (Fig. 3C). These postsynaptic responses were mostly mediated by AMPARs because they were effectively blocked (by $>95 \%$ ) with the AMPAR antagonist NBQX (Fig. 3C). Together, the results from these two experiments indicate that the inhibitory effect of H-1152 on AMPAergic transmission is not attributable to changes in the postsynaptic sensitivity to glutamate, because any such change should be manifested as changes in the amplitudes of mEPSCs AMPA $_{\text {and whole- }}$ cell responses to exogenous glutamate pulses.

In light of these previous results, we considered the possibility that depression of excitatory synaptic responses by ROCK inhibition in the HN was due to a presynaptic mechanism. We tested this possibility by recording spontaneous AMPAergic synaptic currents under conditions of facilitated spontaneous glutamate 
release $\left(\mathrm{sEPSCs}_{\mathrm{AMPA}}\right)$. Under these pharmacological conditions (see Materials and Methods), we would expect that spontaneous synaptic activity was a mixture of action potential-dependent and -independent events. Consistent with this prediction, sEPSCs $s_{\text {AMPA }}$ had peak amplitudes and frequencies 1.42- and 1.75-fold higher, respectively, than those calculated for IEPSC $_{\text {AMPA }}$. We therefore examined the effect of H-1152 on sEPSCs AMPA. Under $_{\text {AM }}$ control pretreatment conditions sEPSC$\mathrm{S}_{\text {AMPA }}$ had a mean amplitude of $33.9 \pm$ $2.4 \mathrm{pA}$, and a variable frequency ranging from 4.6 to $17.4 \mathrm{~Hz}(11.9 \pm 2.3 \mathrm{~Hz})$; after H-1152 treatment, the mean sEPSCs $s_{\text {AMPA }}$ amplitude was significantly reduced to $74.0 \%(25.1 \pm 1.1 \mathrm{pA})$ (Fig. $4 A, B)$. Consequently, the cumulative distribution of SEPSC $_{\text {AMPA }}$ amplitude was significantly shifted to the left, indicating lower event amplitudes after treatment $(p<0.01$; KolmogorovSmirnov test) (Fig. 4C). The attenuation reached steady state within $5 \mathrm{~min}$, and sEPSC $_{\text {AMPA }}$ amplitude recovered after 5 min of washout (Fig. $4 A-C$ ). Although we found a consistent tendency in the frequency of $s$ EPSC $_{\text {AMPA }}$ to decrease after $\mathrm{H}-1152$ application $(11.9 \pm 2.3 \mathrm{~Hz}$ before vs $9.6 \pm 2.3 \mathrm{~Hz}$ after $\mathrm{H}-1152)$, these differences did not reach statistical significance ( $p=0.23$, one-way ANOVA for repeated measures). The above results are more consistent with the idea that ROCK inhibition elicits depression of action potential- and $\mathrm{Ca}^{2+}$ dependent glutamatergic transmission through a mechanism involving presynaptic modifications.

To confirm this notion, we examined the effect of $\mathrm{H}-1152$ on facilitation of electrically evoked eEPSCs AMPA $_{\text {using }}$ paired-pulse and repetitive afferent stimulation from the VLRF. Under repetitive stimulation, a change in the amount of facilitation is considered to be attributable to a presynaptic change in the release probability (Zucker, 1989). Thus, synapses with a high release probability tend to produce largeamplitude EPSCs following single-pulse afferent stimulation and exhibit little facilitation upon repetitive stimulation. If the H-1152-mediated synaptic attenuation involved a presynaptic mechanism of action, it would be associated with an increase in the magnitude of facilitation. Under control conditions, eEPSC$\mathrm{s}_{\mathrm{AMPA}}$ evoked by paired stimulation of VFLR afferent fibers displayed a robust facilitation over the entire range of interstimulus intervals (Fig. 4D,E). However, facilitation was typically more pronounced at shorter interstimulus intervals and smaller at longer intervals. The mean paired-pulse facilitation ratio (PPR) was $1.89 \pm$ 0.12 with an interval of $25 \mathrm{~ms}$ and $1.65 \pm 0.08$ at $50 \mathrm{~ms}$, decreasing to $1.39 \pm 0.06$ at $100 \mathrm{~ms}$ and to $1.18 \pm 0.04 \mathrm{~ms}$ at $200 \mathrm{~ms}$ intervals. After 5 min application of $\mathrm{H}-1152$, paired-pulse facilitation showed a marked and reversible increase at 50 and $25 \mathrm{~ms}$ intervals (Fig. 4E). On average, the magnitude of PPR increased by $14.4 \%$ at $25 \mathrm{~ms}$ (mean $2.15 \pm 0.16$ ) and by $10.9 \%$ at $50 \mathrm{~ms}$ interval (mean $1.82 \pm$ 0.12) after H-1152 application.

Repeated VLRF stimulation with a train of 20 shocks at $40 \mathrm{~Hz}$ also resulted in a powerful facilitation of eEPSCs ${ }_{\mathrm{AMPA}}$, with a progressive enhancement in the eEPSCs ${ }_{\mathrm{AMPA}}$ amplitude through the train (Fig. 4F, G). The standard facilitation index was calculated by measuring the amplitude of the individual eEPSCs ${ }_{\mathrm{AMPA}}$ throughout the train in relation to the first $\mathrm{eEPSC}_{\mathrm{AMPA}}$. In control recordings, the average values of the facilitation index for the 10 th and 20 th eEPSCs AMPA $_{\text {were }} 1.79 \pm 0.19$ and $1.80 \pm 0.23$, respectively. Application of $\mathrm{H}-1152$ to the bath reversibly potentiated this facilitation in all HMNs tested (Fig. $4 F, G$ ). Thus, after H-1152 the facilitatory index for the 10th eEPSCs $s_{\text {AMPA }}$ was $2.51 \pm 0.18$, whereas for the 20th eEPSCs AMPA $_{\text {this value was }}$ $2.59 \pm 0.18$. The increase in the magnitude of the facilitatory index induced by $\mathrm{H}-1152$ was statistically significant from the

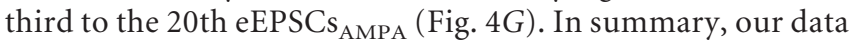
from paired-pulse and train stimulation tests demonstrate that the reduction in the eEPSCs${ }_{\mathrm{AMPA}}$ amplitude by endogenous ROCK inhibition was accompanied by an increase in the magnitude of facilitation induced by repetitive afferent stimulation.

The preceding results show that the attenuation of the evoked AMPAergic responses induced by ROCK inhibition was related to a reduction in the glutamate release probability and to an increase in the amount of facilitation. Release probability at single central synapses is believed to be determined by the number of fusion-competent vesicles (Dobrunz, 2002; Millar et al., 2002). Therefore, changes in synaptic strength induced by ROCK inhibition could reflect a decrease in the size of the readily releasable pool (RRP) of synaptic vesicles. This idea was further strengthened by the subsequent analysis of eEPSCs AMPA $_{\text {amplitudes using }}$ a minimal stimulation paradigm, which is designed to stimulate only one fiber and a single or small number of release sites. Typically, the intensity of the stimulation was set to elicit eEPSCs $_{\text {AMPA }}$ with $\sim 30 \%$ to $40 \%$ failures. Under control conditions, eEPSCs $s_{\text {AMPA }}$ amplitudes evoked by minimal stimulation were distributed over a range from zero to $\sim 80 \mathrm{pA}$ (Fig. $4 \mathrm{H}$ ), with a mean amplitude of $40.0 \pm 3.1 \mathrm{pA}(n=3 \mathrm{HMNs}$, excluding 
A

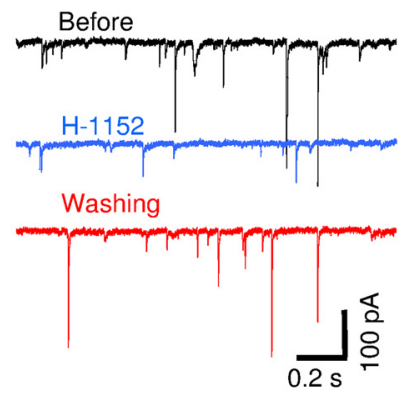

B

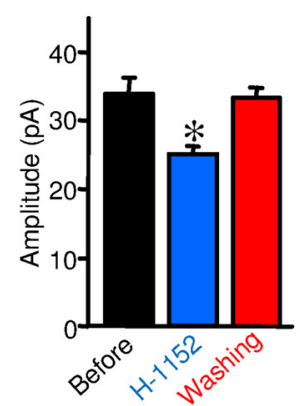

C

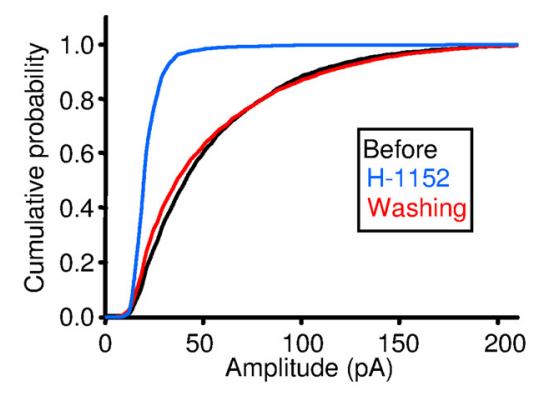

D

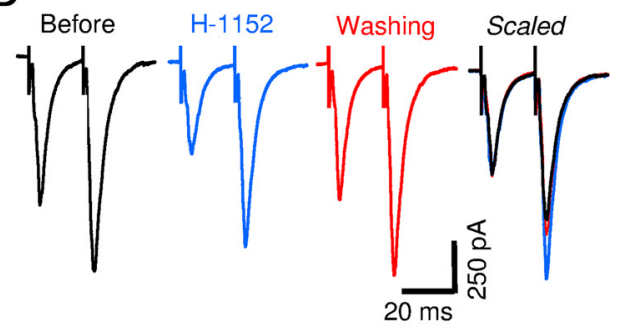

F

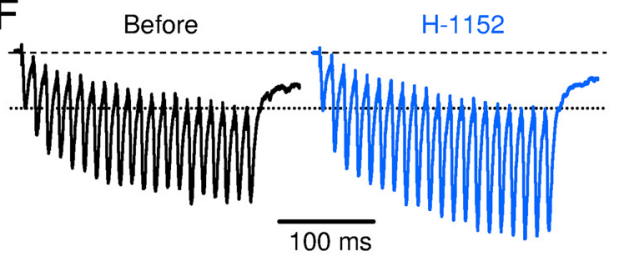

H

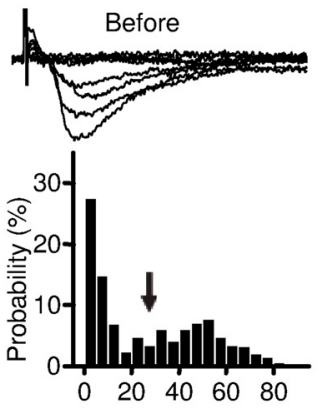

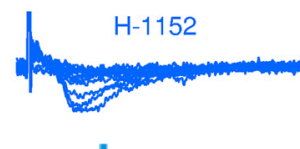

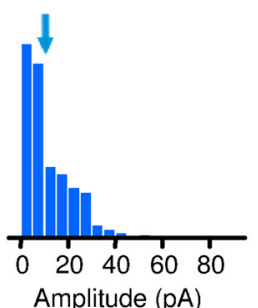

E

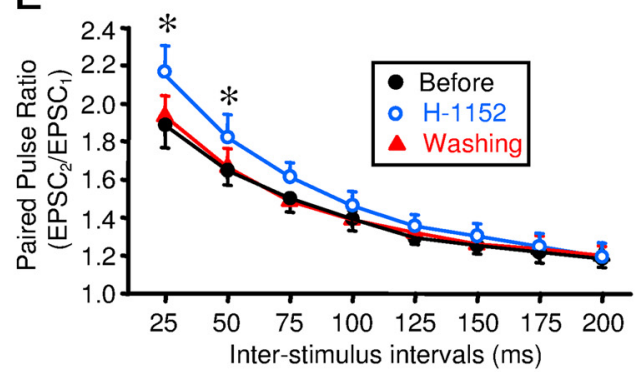

G

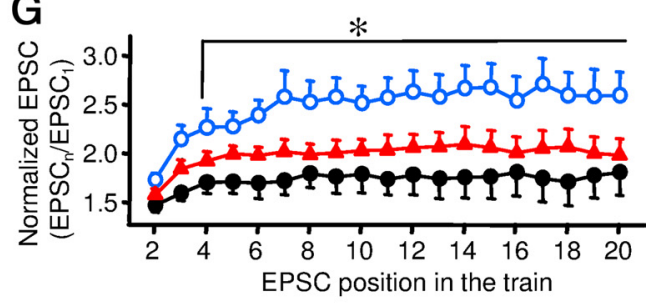

I
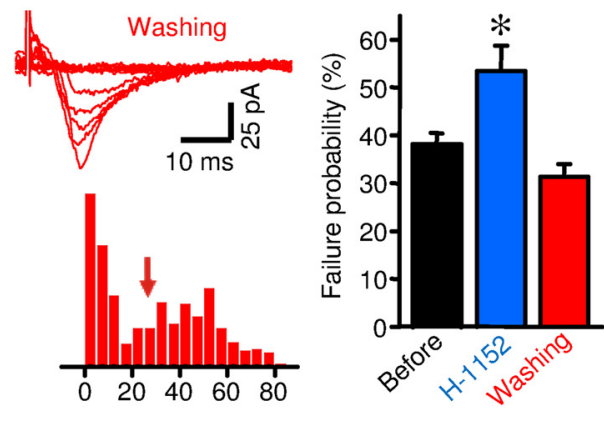

Figure 4. The mechanism underlying ROCK-dependent maintenance of synaptic strength operates presynaptically. A, Current traces of sEPSCS $S_{\text {AMPA }}$ recorded from an HMN before (black), following treatment with H-1152 (blue), and after washing (red). sEPSCS AMPA $_{\text {a }}$ were isolated in the presence of bicuculline, strychnine, APV, and D-tubocurarine and recorded at $-65 \mathrm{mV}$ in a high-K ${ }^{+}$, high- $\mathrm{Ca}^{2+}$ containing aCSF without tetrodotoxin. B, Average $\mathrm{SEPSC}_{\mathrm{AMPA}}$ amplitude for the H-1152-treated group of HMNs compared with their respective pretreatment (control) and washout periods $(n=6 \mathrm{HMNs}) .{ }^{*} p<0.05$, one-way ANOVA for repeated measures. $C$, Normalized cumulative probability distributions of $s E P S C_{\text {AMPA }}$ amplitudes measured for each condition (listed in the inset box). Bin width, $2 \mathrm{pA}$. D, Examples of eEPS(S $S_{\text {AMPA }}$ recorded in response to paired-pulse stimulation of VLRF axons in an HMN before (black) and after treatment with H-1152 (blue), and

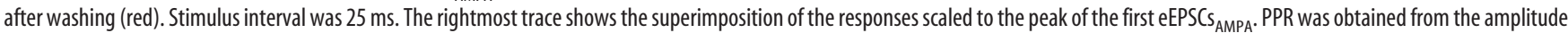

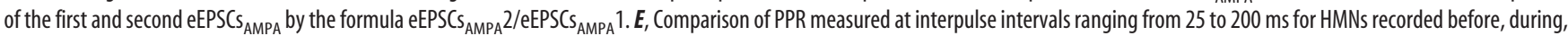
and after washout of the drug $(n=5 \mathrm{HMNs})$. ${ }^{*} p<0.05$, two-way ANOVA for repeated measures. $\boldsymbol{F}$, Sample records illustrating succession of eEPSC $s_{\text {AMPA }}$ in an HMN evoked by a train of 20 stimuli

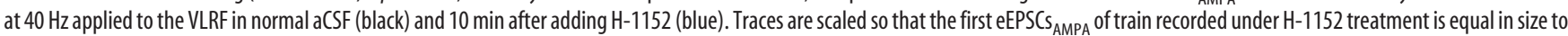

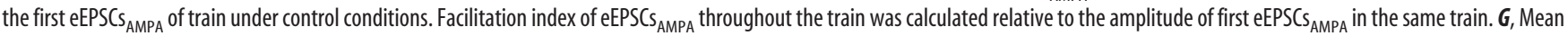
eEPSCS $S_{A M P A}$ amplitude, normalized to the first eEPSCS $S_{A M P A}\left(e E P S C S_{A M P A} n / e E P S C S_{A M P A} 1\right)$ and plotted against the position number of eEPSC $S_{A M P A} n$ within the train (1-20) for the $H-1152$-treated group of HMNs under control, drug perfusion, and washout conditions $(n=6 \mathrm{HMNs})$. Symbol code listed in $\boldsymbol{E}$, above. ${ }^{*} p<0.05$, two-way ANOVA for repeated measures. $\boldsymbol{H}$, Top, Representative examples showing superimposition of 10 successive eEPSCS $\mathrm{S}_{\mathrm{AMPA}}$ evoked at $0.2 \mathrm{~Hz}$ by minimal stimulation of VLRF in an HMN before (black), after treatment with $\mathrm{H}-1152$ (blue) and after washing (red). Bottom, Amplitude distribution histograms of eEPSCS ${ }_{A M P A}$ in response to minimal stimulation before, during, and after $\mathrm{H}-1152$ treatment. Arrows indicate mean values. Note the reversible shift of the distribution to the left under $\mathrm{H}$-1152. Each histogram is made of 600 responses ( $5 \mathrm{pA}$ bin size) pooled from $3 \mathrm{HMNs}$. I, Histogram showing changes in eEPSC $S_{\text {AMPA }}$ failure rates to minimal stimulation for the control, H-1152, and washout conditions. The fraction of events classified as failures was significantly increased by H-1152 $(n=3)$. ${ }^{*} p<0.05$, one-way ANOVA on ranks for repeated measures. 
failures). This value is close to the mean $\mathrm{sEPSC}_{\mathrm{AMPA}}$ amplitude and almost double the average amplitude of mEPSCs $_{\mathrm{AMPA}}$, a difference that could be interpreted as the activation of a single afferent fiber causing the release of more than one vesicle at the same or different VLRF-HMN contact sites. As summarized in Figure $4 \mathrm{H}$, bath application of $\mathrm{H}-1152$ significantly reduced the peak amplitude of eEPSCs ${ }_{\text {AMPA }}$ obtained with minimal stimulation (from $40.0 \pm 3.1 \mathrm{pA}$ to $18.3 \pm 0.9 \mathrm{pA}$, excluding failures), an effect that was accompanied by marked narrowing of the amplitude distribution toward lower amplitudes (upper limit of $\sim 55$ $\mathrm{pA})$ and by an increase of the eEPSCs $\mathrm{AMPA}_{\mathrm{A}}$ failure rate (38.4 \pm $2.4 \%$ before vs $53.6 \pm 5.5 \%$ after $\mathrm{H}-1152$; Fig. $4 H, I)$. All these effects were reversible with washing (Fig. $4 H, I$ ).

Together, these data strongly suggest that the depression of synaptic strength induced by ROCK inhibition is completely dependent on a reduction in the release probability from excitatory glutamatergic terminals apposed on HMNs, an effect probably attributable to a reduction in the RRP of synaptic vesicles. These results indeed demonstrate that endogenous ROCK signaling maintains normal excitatory synaptic transmission through a purely presynaptic mechanism.

\section{The presynaptic mechanism underlying the ROCK-dependent maintenance of synaptic strength involves MLCK inhibition, presumably by PAK phosphorylation}

Our previous results show that basal activity of presynaptic ROCK in the HN is required to maintain evoked glutamate release from excitatory afferent terminals at optimal levels. ROCK activation usually results in MLC phosphorylation, by a direct action on MLC and/or by inhibition of MLCP. Phosphorylation of MLC subsequently induces stimulation of actinmyosin interactions, as reviewed by Luo (2002); however, our immunoblot analysis on $\mathrm{HN}$ homogenates unexpectedly revealed an increase $(40.8 \pm 15.1 \%)$ in the ratio of p-MLC to MLC in H-1152-incubated relative to aCSF-incubated brainstem slices (Fig. $5 A, B$ ).

Since the level of p-MLC is regulated by the balanced actions of MLCK and MLCP (Luo, 2002), our results led us to consider the possibility that endogenous ROCK may have an inhibitory effect on MLCK. To test this idea, we used ML-7, a specific inhibitor of MLCK, in Western blot and electrophysiological experiments. Incubation of neonatal brainstem slices with ML-7 for 10 min had no effects on the p-MLC/MLC ratio (Fig. $5 A, B$ ), thereby indicating the absence of a net action of endogenous MLCK on the levels of MLC phosphorylation. However, the H-1152induced increase in p-MLC was fully prevented by coaddition to the bath of the MLCK inhibitor (Fig. 5A,B). In agreement with these results, the electrophysiological recordings show that ML-7 alone did not alter ( $p=0.60$, paired $t$ test, $n=5 \mathrm{HMNs}$ ) the mean peak amplitude of eEPSCs AMPA $_{\text {evoked in HMNs by electrical }}$ stimulation of VLRF $(-727.1 \pm 178.2 \mathrm{pA}$ before vs $-709.4 \pm$ $153.7 \mathrm{pA}$ after ML-7), but fully reverted the reduction induced by H-1152 when ML-7 was subsequently added to the bath $(-684.4 \pm 109.9 \mathrm{pA}$ before, $-465.6 \pm 81.3 \mathrm{pA}$ after $\mathrm{H}-1152$, and $622.4 \pm 105.6 \mathrm{pA}$ after ML-7) (Fig. 5C,D). Likewise, ML-7 re-

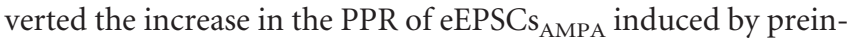
cubation with $\mathrm{H}-1152$, which provides support for a presynaptic site of ML-7 action (1.55 \pm 0.07 before and $2.40 \pm 0.18$ after $\mathrm{H}-1152$, and $1.56 \pm 0.06$ after ML-7) (Fig. $5 E, F$ ). Together, the preceding data point to MLCK as a main molecular substrate being directly or indirectly inhibited by endogenously active ROCK within excitatory presynaptic terminals. This mechanism, in turn, keeps baseline levels of MLC phosphorylation and facil- itates excitatory synaptic transmission, at least in our experimental conditions (see Fig. 9 for a schematic representation of the proposed molecular pathway).

Given that evidence for direct inhibition of MLCK by ROCK has not been reported so far, we next looked for a molecular partner that could act downstream of ROCK with inhibitory properties on MLCK. A candidate molecule for mediating such action is p21-activated kinase (PAK), which catalyzes an inhibitory phosphorylation of MLCK and indirectly reduces MLC phosphorylation (see Fig. 9) (Li et al., 2010). Interestingly, Western blot analysis of $\mathrm{HN}$ homogenates revealed that the level of $\mathrm{PAK}_{1-3}$ phosphorylation was strongly reduced by treatment with the ROCK inhibitor H-1152 $(-48.2 \pm 15.5 \% ; p<0.05$, Mann-Whitney $U$ test; $n=3$ independent experiments) (Fig. $5 G$ ). This finding suggests a novel molecular mechanism by which basally active ROCK could inhibit actomyosin contractility (see Fig. 9).

\section{Inhibition of endogenous ROCK reduces the size of the RRP of synaptic vesicles in a MLCK-dependent way}

Before investigating the final effector mechanism underlying the $\mathrm{H}$-1152-mediated attenuation of glutamate release during spontaneous or evoked neurotransmission, we assessed the expression of ROCK protein in glutamatergic presynaptic terminals within the HN from neonatal rats by immunohistochemistry. To that end, we performed triple immunofluorescence staining with antibodies against ROCK $\alpha-\beta$, VGLUT2, and SMI32. It is important to clarify that labeling of HMN somata almost fully disappeared when the anti-ROCK $\alpha$ antibody dilution was decreased to half (1:100), suggesting a higher concentration of the antigen in puncta-like structures than in motoneuron perikarya. Immunohistochemistry against ROCK $\alpha$ was performed at this dilution to minimize interference with perikarya immunoreactivity. ROCK-ir punctate elements were frequently observed either in close proximity to or partially colocalizing with VGLUT2-ir puncta apposed to HMNs (Fig. 6A,B). This distribution pattern is consistent with the presumed location of ROCK at the central core of the presynaptic boutons, from where it exerts a regulatory role on cytoskeleton dynamics and microtubule organization (Benarroch, 2007). Our quantitative analysis revealed that two thirds ( $66.6 \pm 4.5 \% ; n=15 \mathrm{HMNs}$ ) of VGLUT2-ir puncta apposed to the perikarya of HMNs showed a direct relationship with ROCK-positive structures (Fig. 6A,B). These structural data are in agreement with our electrophysiological results and support the notion that most glutamatergic afferent inputs on HMNs contain the molecular machinery required for a presynaptic action of ROCK.

At this point, we hypothesized that endogenous ROCK signaling might, through its modulatory control on the actin-myosin cytoskeleton, regulate clustering and spatial distribution of synaptic vesicle pools within excitatory presynaptic boutons. Therefore, we analyzed the ultrastructural organization of excitatory synaptic boutons on HMNs in control and treated neonatal brainstem slices by electron microscopy. Under electron microscopy, HMNs are easily distinguishable from interneurons, which present a noticeable invaginated nucleus and smaller soma sizes in cross-sectional areas at the nucleolar level (Sunico et al., 2011). As we have reported (Sunico et al., 2010), presynaptic boutons on the somatic membrane of HMNs with a presumably excitatory function were identified by the spherical nature of their synaptic vesicles, assisted by synaptic densities when these were evident, and classified as S-type boutons. To quantify the spatial distribu- 

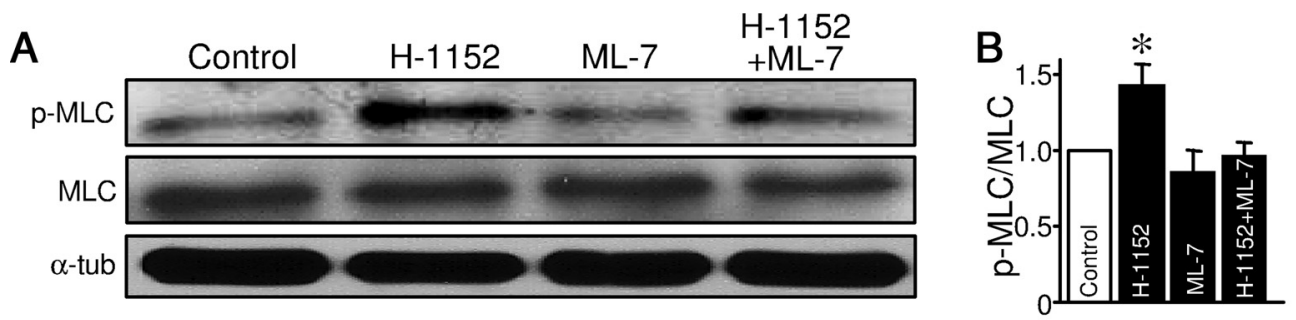

C

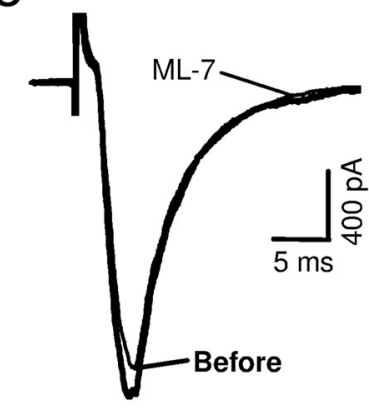

$\mathrm{E}$

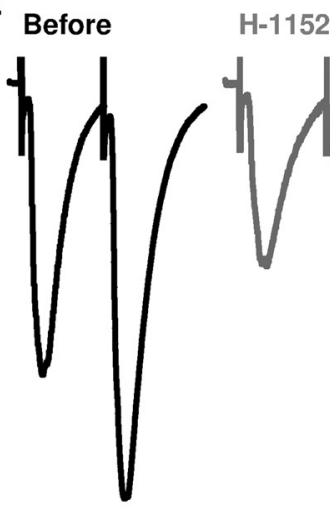

$\mathrm{H}-1152$

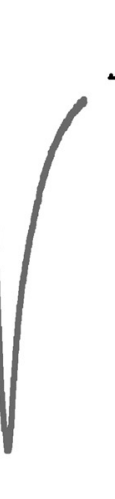

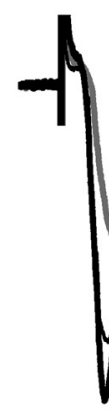

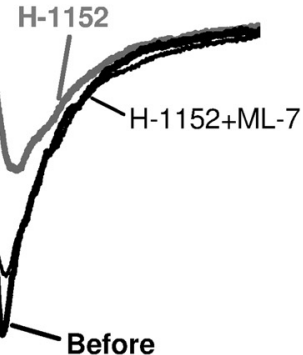

Before $\mathrm{H}-1152+\mathrm{ML}-7$

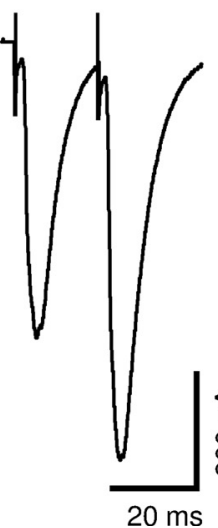

D

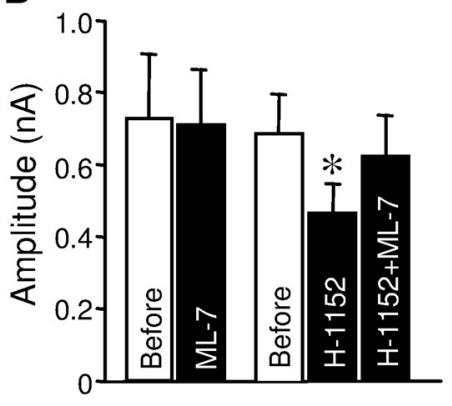

F

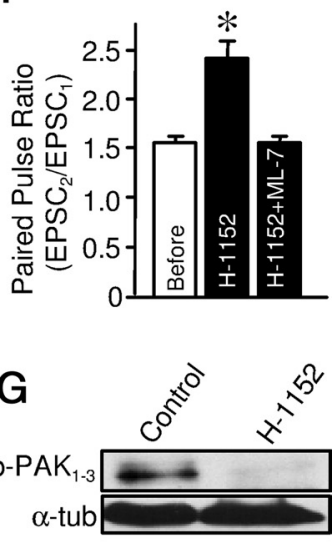

Figure 5. Inhibition of endogenous ROCKactivity increases p-MLC levels in the HN via a mechanism that involves MLCK activation and is accompanied by PAK dephosphorylation. $A$, Western blot of phosphorylated and total MLC protein levels (denoted as p-MLC and MLC, respectively) in the HN of neonatal brainstem slices incubated for 10 min in aCSF alone (control) or supplemented with either H-1152, $10 \mu \mathrm{m} \mathrm{ML-7}$ or with H-1152 plus ML-7. $\alpha$-Tubulin ( $\alpha$-tub) expression was used as an internal loading reference. $\boldsymbol{B}$, Histogram showing the average ratio of $\mathrm{p}$-MLC to total MLC densitometric intensity for the control and treated slices. Ratio values were normalized relative to the control group. Columns represent average of at least four independent experiments. ${ }^{*} p<0.05$, one-way ANOVA on ranks, post hoc Dunn's test. C, Left traces, Examples of eEPSCS AMPA $_{\text {P }}$ recorded from an HMN in normal aCSF (control; thick black) and after 10 min bath perfusion with ML-7 (thin black line). Right traces, eEPSCS AMPA $_{1}$ recorded in an HMN before (thick black) and sequentially after adding to the bath H-1152 (gray) and then ML-7 (thin black line). D, Histogram showing quantitative comparison of average EPSC $_{\text {AMPA }}$ amplitude for the ML-7 $(n=5)$ and H-1152 plus ML-7 $(n=4)$ treated groups of HMNs compared with their respective pretreatment controls (before).

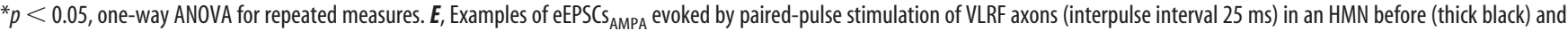
following treatment with H-1152 (gray) and finally after coaddition of ML-7 (thin black). The rightmost trace shows the superimposition of the responses scaled to the peak of the first eEPS $\mathrm{Cs}_{\mathrm{AMPA}}$.

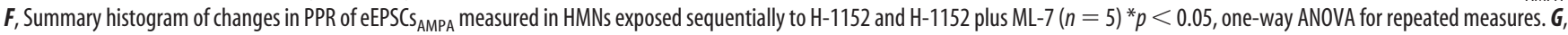
Western blot analysis of $\mathrm{p}-\mathrm{PAK}_{1-3}$ in $\mathrm{HN}$ homogenates from control and $\mathrm{H}$-1152-treated brainstem slices. $\alpha$-tub was used as loading reference.

tion of vesicles relative to the a.z. within S-type boutons, three regions were delimited by lines parallel to the a.z. profile, and we counted the number of vesicles included in the intervals of $0-100$ $\mathrm{nm}, 100-200 \mathrm{~nm}$, and 200-300 nm away a.z. (Fig. 6C). For each bouton, we also counted all vesicles in the entire vesicle pool to evaluate feasible effects on vesicle trafficking and/or recycling (Fig. 6D). Visualization of high-resolution images of S-type boutons attached to HMNs in $\mathrm{H}-1152$-treated slices revealed a conspicuous reduction in the number of vesicles near the a.z. (Fig. $6 E$ ). In slices incubated in aCSF (control), the number of vesicles averaged $9.6 \pm 0.4$ in the interval nearest the a.z., $9.7 \pm 0.4$ in the intermediate, and $8.9 \pm 0.6$ in the more distant interval. The mean number of vesicles in the entire pool in the control condition was $53.9 \pm 3.3$ per a.z. $(n=133$ boutons $)$. A significant reduction in the number of vesicles was observed in the two regions closest to the a.z. after $\mathrm{H}-1152$ treatment $(0-100 \mathrm{~nm}$, $-16.7 \pm 3.7 \%$; $100-200 \mathrm{~nm},-13.8 \pm 4.3 \%$ ) (Fig. $6 \mathrm{~F}$ ), along with a nonsignificant $(-8.9 \pm 5.7 \%)$ reduction in the more distant region (Fig. $6 E, F$ ). These changes in the size of the vesicle pool were prevented by coincubation with the MCLK inhibitor ML-7 (Fig. 6E, F). Finally, we measured the linear density (number of vesicles per $100 \mathrm{~nm}$ membrane) of synaptic vesicles docked at the a.z. (Fig. $6 G$ ). Addition to the bath of the ROCK inhibitor induced a significant reduction $(-20.9 \pm 6.4 \%)$ in the number of docked vesicles; coincubation with ML-7 prevented this reduction $(-1.9 \pm 6.6 \%)$. These outcomes suggest that endogenous ROCK signaling may be involved in the maintenance of the RRP of synaptic vesicles. Interestingly, none of the pharmacologic 

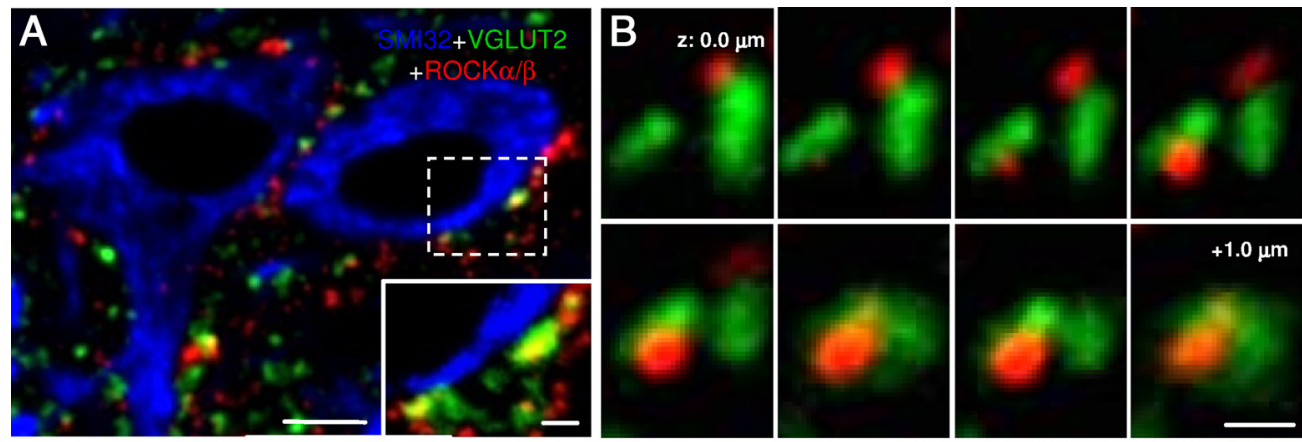

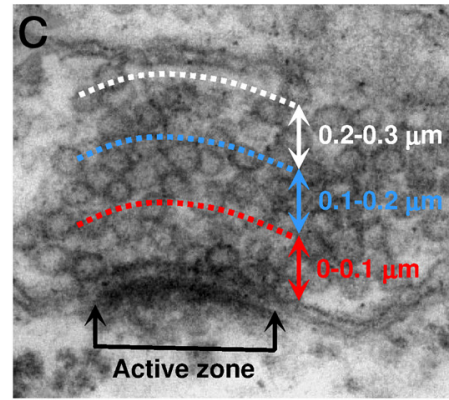

$\mathrm{F}$

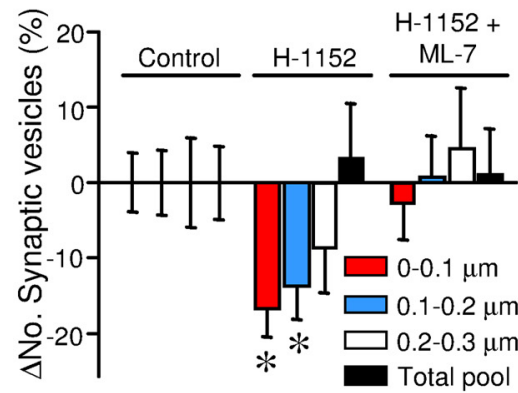

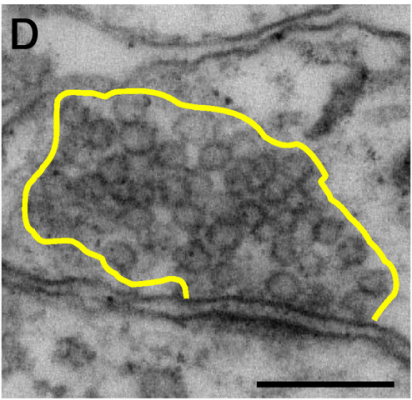

G
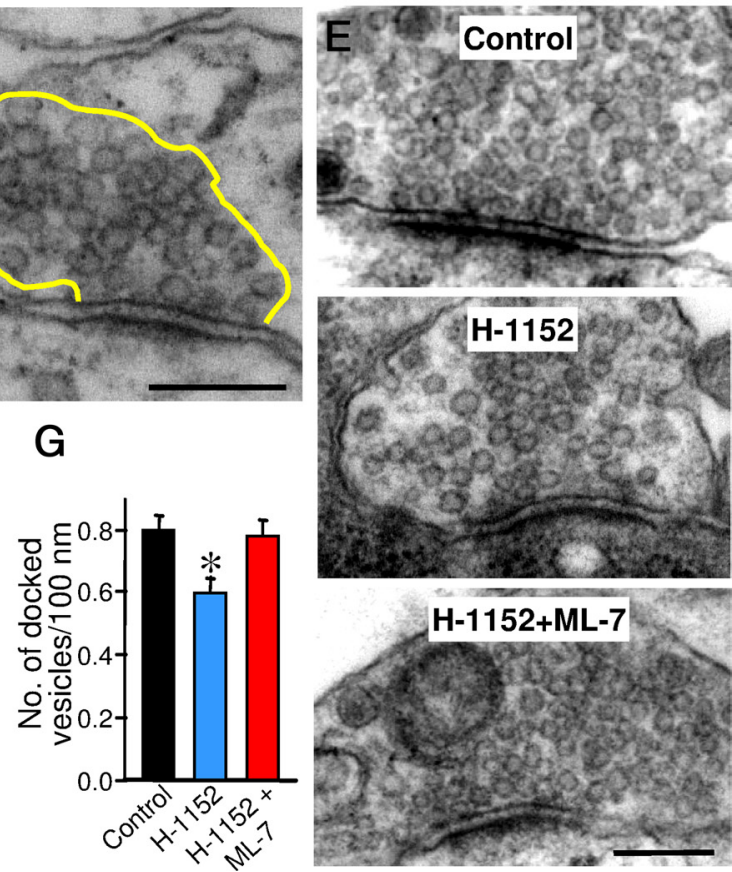

Figure 6. ROCKinhibition reduces the size of the RRP of synaptic vesicles through a mechanism that involves MLCK activation. $\boldsymbol{A}$, Triple-labeling confocal immunofluorescence for the motoneuron marker SMI32 (blue), the vesicular glutamate transporter 2 (VGLUT2; green), and ROCK ( $\alpha$ and $\beta$ isoforms; red) in a high-magnification image captured from a selected region of the HN from a neonatal (P7) rat. Yellow signals indicate the sites of colocalization of VGLUT2 and ROCK $\alpha / \beta$. ROCK-ir clusters were found to be spatially contiguous or colocalizing with VGLUT2-ir terminals. Inset shows at higher magnification a z-stack reconstruction through the dashed box region. $\boldsymbol{B}$, High-magnification confocal z-series showing straight relationship (top panels) between VGLUT2- and ROCK $\alpha / \beta$-ir puncta and partial colocalization in the bottom panels. Scale bars: $\boldsymbol{A}, 25 \mu \mathrm{m}$; inset in $\boldsymbol{A}, 2 \mu \mathrm{m} ; \boldsymbol{B}, 1 \mu \mathrm{m}$. $\boldsymbol{C}, \boldsymbol{D}$, Electron micrographs of two $\boldsymbol{S}$-type boutons (containing spherical vesicles) with asymmetric synaptic contacts on the somatic membrane of an HMN depicting details of the procedure used to examine topographically the numerical changes in synaptic vesicles. The number of synaptic vesicles was counted in three zones, each $0.1 \mu \mathrm{m}$ wide, parallel to the membrane of the synaptic cleft and at successively greater distances from the a.z. (C). The first region (boxed with a red dotted line) encloses an area directly adjacent to the a.z. membrane. The intermediate region (boxed with a blue dotted line) was located in the interval from $0.1 \mu \mathrm{m}$ to $0.2 \mu \mathrm{m}$ away from the a.z. Finally, the more distant region (boxed with a white dotted line) occupied an area corresponding to the distance interval from $0.2 \mu \mathrm{m}$ to $0.3 \mu \mathrm{m}$. The total number of synaptic vesicles contained in each bouton was also quantified (D). Scale bar, $200 \mathrm{~nm}$. $\boldsymbol{E}$, Electron microscopy images of S-type boutons over the soma of HMNs from neonatal rats following incubation of brainstem slices in aCSF alone (control) or supplemented with either $\mathrm{H}-1152$ or with $\mathrm{H}-1152$ plus ML-7. Scale bar: $200 \mathrm{~nm}$ (applies to all panels). $\boldsymbol{F}$, Quantitative changes in the number of synaptic vesicles (expressed as percentage change from control) contained in each spatial compartment after treatment with the indicated drugs. Histogram bins indicate distances from the a.z. as indicated in the legend. $\mathbf{G}$, Histogram showing the linear density of docked synaptic vesicles per $100 \mathrm{~nm}$ a.z. under the indicated conditions. * $p<0.05$, one-way ANOVA, post hoc Tukey's test.

Table 1. Ultrastructural characterization of S-type boutons attached to HMNs

\begin{tabular}{|c|c|c|c|c|c|}
\hline & Length of a.z. $(\mu \mathrm{m})$ & No. of a.z. per bouton & Bouton area $\left(\mu \mathrm{m}^{2}\right)$ & HMN perimeter $(\mu \mathrm{m})$ & No. of boutons/HMN \\
\hline Control & $0.24 \pm 0.08(169)$ & $1.27 \pm 0.05(133)$ & $0.35 \pm 0.02(131)$ & $70.9 \pm 2.3(50)$ & $2.8 \pm 0.3(86)$ \\
\hline H-1152 & $0.25 \pm 0.07(119)$ & $1.21 \pm 0.04(102)$ & $0.44 \pm 0.02(100)^{*}$ & $70.7 \pm 2.5(32)$ & $2.5 \pm 0.5(35)$ \\
\hline H-1152 + ML-7 & $0.24 \pm 0.09(140)$ & $1.22 \pm 0.05(115)$ & $0.32 \pm 0.02(110)$ & $75.4 \pm 1.9(30)$ & $2.3 \pm 0.2(103)$ \\
\hline
\end{tabular}

Number of sampled motoneurons, boutons or a.z. is indicated in parentheses. ${ }^{*} p<0.05$, one-way ANOVA, post hoc Tukey's test, relative to the control group.

treatments altered the total number of vesicles in the entire vesicle pool (Fig. 6F).

To identify other presynaptic changes that could also contribute to $\mathrm{H}$-1152-induced reduction in release probability from excitatory boutons, we further analyzed the average a.z. length, mean number of a.z. per bouton, average number of excitatory boutons per motoneuron, and mean cross-sectional bouton area
(Table 1). The ultrastructural analysis shows that none of these drug treatments had any significant effect on the first three parameters (Table 1). However, treatment with $\mathrm{H}-1152$ induced an increase in the mean cross-sectional area of S-type boutons; this was fully prevented by coincubation with ML-7 (Table 1). This change in bouton size might presumably reveal a dynamic remodeling of the actin cytoskeleton regulated by the ROCK- 
A

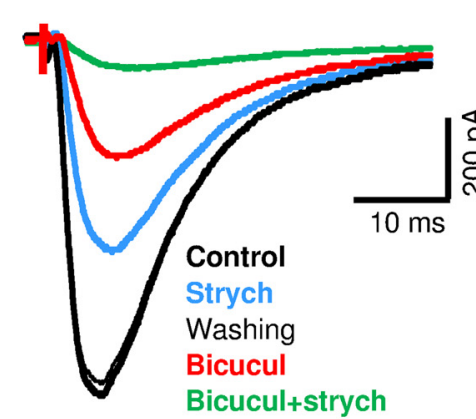

B

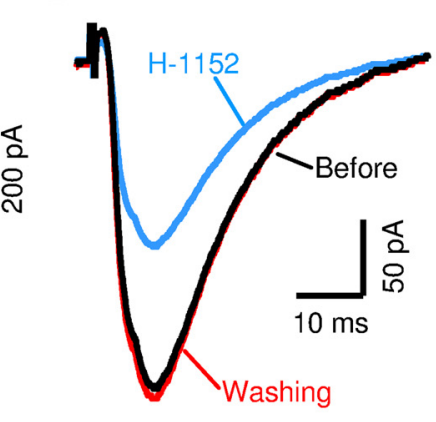

C

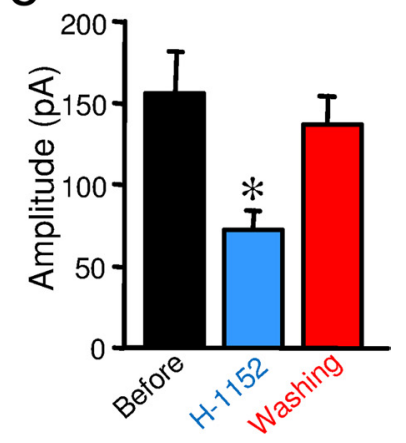

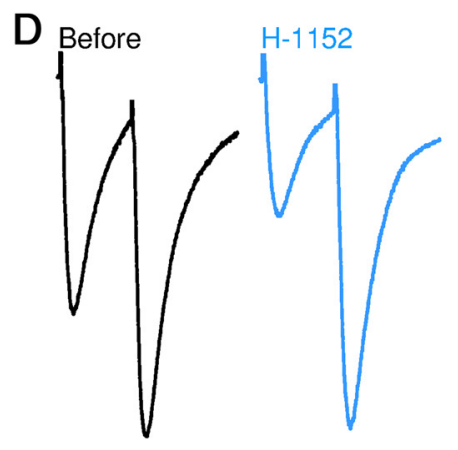
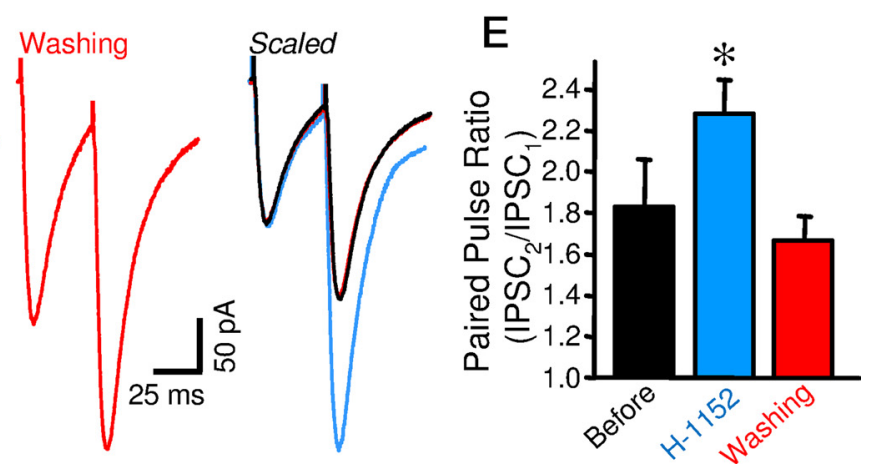

Figure 7. Inhibition of ROCK activity reduces GABAergic transmission by a presynaptic mechanism. $A$, Pharmacological profile of elPSCs in HMNs. To isolate chloride-dependent elPSCs, pipettes were filled with a high-chloride internal solution and recordings were performed in the presence of $20 \mu \mathrm{MNBQX}, 50 \mu \mathrm{M}$ APV, and $30 \mu \mathrm{m} \mathrm{D}$-tubocurarine. elPSCs were evoked from a holding potential of $-70 \mathrm{mV}$ via electrical stimulation of the VLRF and pharmacologically dissected into its composite subtypes. The relative contribution of glycine-versus $G A B A_{A}$-receptor-mediated components to eIPSCs was determined by addition of the selective receptor antagonists strychnine $(1 \mu \mathrm{M})$ and bicuculline (10 $\mu \mathrm{M})$, respectively. Traces show averaged elPSCs (each an average of 10 responses) recorded from an HMN before (thick black) and after superperfusion first with strychnine (blue) and then with control aCSF (wash; thin black), followed by bath application of bicuculline (red), and finally after coaddition of strychnine (green). B, Superimposition of averaged elPSCS $\mathrm{S}_{\mathrm{GABA}}$ from an $\mathrm{HMN}$ recorded before (black), following a 10 min exposure to $\mathrm{H}-1152$ (blue), and after 10 min of

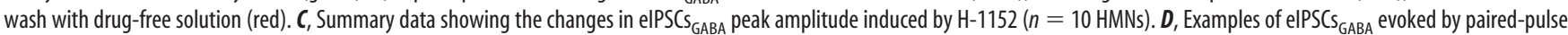
stimulation of VLRF axons (interpulse interval $50 \mathrm{~ms}$ ) in an HMN recorded before (black), during (blue), and after washout of H-1152 (red). The rightmost trace shows the superimposition of the responses scaled to the peak of the first elPSCS ${ }_{G A B A}$. E, Summary histogram of changes in PPR of elPSCs ${ }_{G A B A}$ induced by H-1152 $(n=10 \mathrm{HMNs})$. ${ }^{*} p<0.05$, one-way ANOVA for repeated measures.

MLCK signaling pathway. In conclusion, these data indicate that the attenuation of glutamatergic transmission on HMNs induced by suppression of endogenous ROCK activity is the consequence of a redistribution of the vesicle pool within the terminal rather than a disruption of vesicle trafficking and/or recycling, which affects the RRP of synaptic vesicles (see Fig. 9).

\section{Inhibition of ROCK activity reduces the strength of} inhibitory synapses on HMNs via a presynaptic mechanism An intriguing question that emerges from the results presented so far is whether this ROCK-dependent mechanism regulating synaptic strength is present only at excitatory glutamatergic synapses or also operates for other neurotransmitter systems. This is particularly relevant with respect to the mechanism's effects on the excitatory/inhibitory balance and on the levels of electrical activity within neuronal circuits. To address this question, we tested the effect of H-1152 on electrically evoked IPSCs (eIPSCs) in HMNs. To maximize eIPSCs generated by the opening of chloride-selective channels (glycine and $\mathrm{GABA}_{\mathrm{A}}$ receptors), HMNs were held at $-70 \mathrm{mV}$ and recorded with a high-chloride pipette solution. Under these recording conditions, the reversal potential calculated by the Nernst equation for a chloride selective conductance was close to $0 \mathrm{mV}$. Mixed GABAergic/glycinergic IPSCs were pharmacologically isolated with AMPA, NMDA, and nicotinic receptor antagonists and detected as inward currents.

To analyze the relative contribution of glycine- and $\mathrm{GABA}_{\mathrm{A}}$ receptor-mediated components to mixed eIPSCs in HMNs, we used the selective antagonists strychnine and bicuculline, respectively (Fig. 7A). In the three cells tested, the strychnine-sensitive component of the eIPSC was significantly smaller than the bicuculline-sensitive component, accounting for $\sim 34 \%$ and $66 \%$ respectively, of the total peak amplitude. This supports a dominant role of GABA in evoked inhibitory neurotransmission from VLRF to HMNs. Therefore, we focused our analysis on the effect of ROCK inhibition on the GABAergic component of the eIPSCs. On average, $\mathrm{H}-1152$ reduced the eIPSCs ${ }_{\mathrm{GABA}}$ amplitude to $46 \%$ of control $(-155.8 \pm 25.5 \mathrm{pA}$ before vs $72.1 \pm 12.0 \mathrm{pA}$ after $\mathrm{H}-1152, n=10 \mathrm{HMNs}$ ) (Fig. $7 \mathrm{~B}, C$ ). To investigate whether the $\mathrm{H}-1152-$ mediated depression of GABAergic transmission involves a presynaptic mechanism, we examined the effect of H-1152 on facilitation of electrically evoked eIPSCs $s_{\text {GABA }}$ using paired-pulse stimulation from the VLRF. As with the evoked glutamatergic currents, paired-pulse stimulation with an interstimulus interval of $50 \mathrm{~ms}$ resulted in amplitude enhancement of the second eIPSC $_{\mathrm{GABA}}$. Bath application of $\mathrm{H}-1152$ significantly increased the mean PPR from $1.82 \pm 0.23$ to $2.28 \pm 0.16(n=10$ HMNs) (Fig. $7 D, E$ ). All changes induced by $\mathrm{H}-1152$ were completely reversed after $10 \mathrm{~min}$ of washout with normal aCSF. These results extend our glutamatergic synapse findings, providing evidence that H-1152 also inhibits GABAergic synaptic transmission via a presynaptic locus of action and leaves open the possibility that the mechanism underlying this ROCK-dependent maintenance of synaptic strength may be ubiquitously expressed in different types of synaptic contacts. 

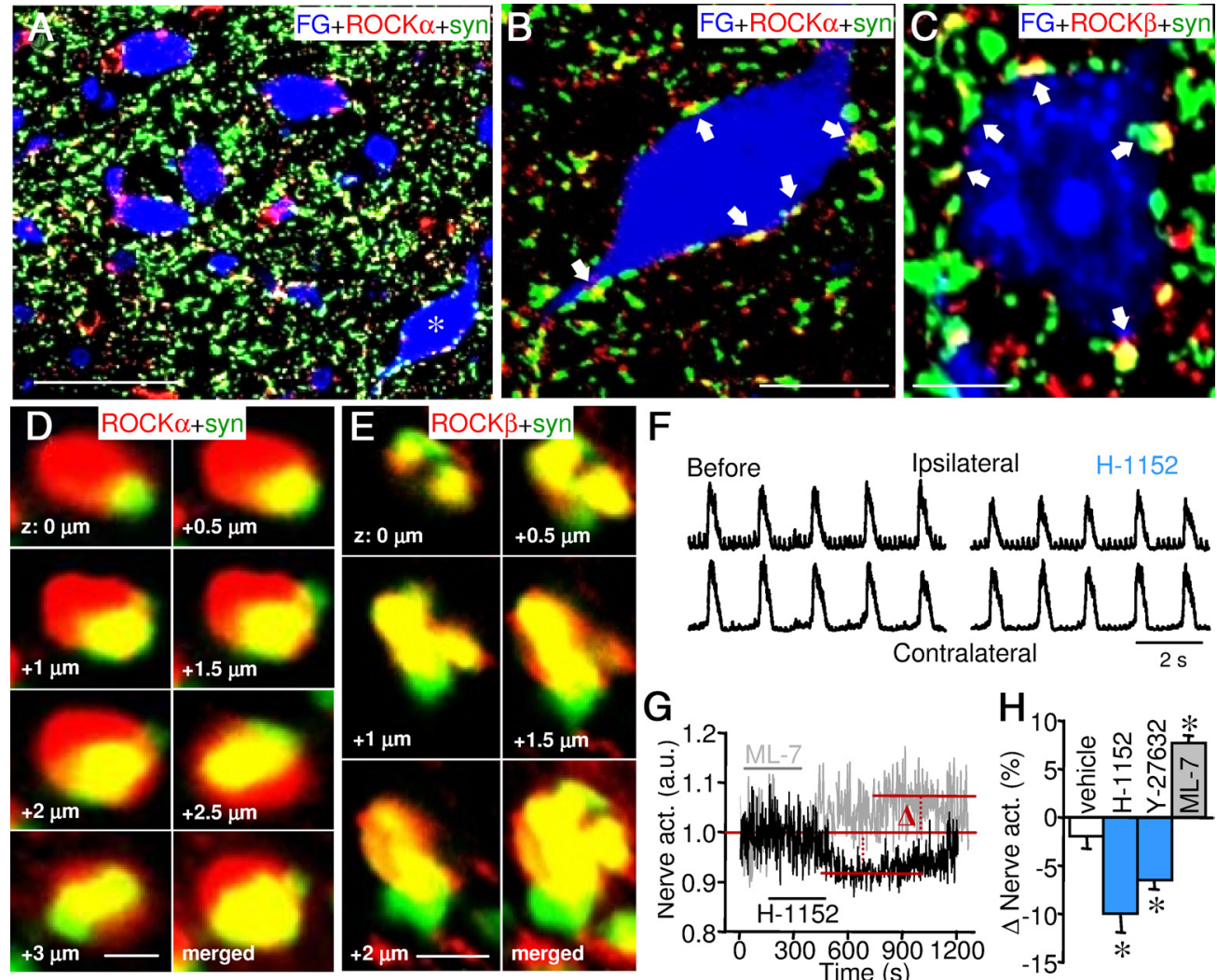

Figure 8. Acute inhibition of endogenous ROCK signaling in the HN attenuates XIIth nerve burst activity in adult rats. $A$, Confocal photomicrograph taken from a selected region of the HN showing dual immunofluorescence for the synaptic marker synaptophysin (syn; green) in combination with ROCK $\alpha$ (red). HMNs were retrogradely labeled with the tongue-injected fluorescent tracer FluoroGold (FG; blue). Asterisk marks motoneuron shown in $\boldsymbol{B}$. Scale bar, $50 \mu \mathrm{m}$. B, C, Confocal images of HMNs retrogradely labeled with $\mathrm{FG}$ in sections double-immunostained for syn and either ROCK $\alpha(\boldsymbol{B})$ or ROCK $\beta(\boldsymbol{C})$. Arrows indicate examples of syn-immunoreactive puncta with colocalized immunofluorescence for each ROCK isoform. Scale bars: $\boldsymbol{B}, 15 \mu \mathrm{m} ; \boldsymbol{C}, 10 \mu \mathrm{m} . \boldsymbol{D}, \boldsymbol{E}$, Highmagnification series of confocal sections collected with z-steps of $0.5 \mu \mathrm{m}$ showing colocalization of ROCK $\alpha(\boldsymbol{D})$ or ROCK $\beta(\boldsymbol{E})$ with syn in presynaptic terminals around HMN cell bodies. The bottom-rightmost panels depict the superposition of all the single confocal sections from each series. Scale bars: $\boldsymbol{D}, 1 \mu \mathrm{m} ; \boldsymbol{E}, 2 \mu \mathrm{m}$. $\boldsymbol{F}$, Integrated Xllth nerve inspiratory activity recorded bilaterally in an anesthetized adult rat before and 5.5 min after unilateral microinjection of $\mathrm{H}-1152$ into the HN. H-1152 ( $2 \mathrm{nmol})$ or Y-27632 ( $5 \mathrm{nmol}$ ) were locally ejected from glass microelectrodes into the HN using pressure microinjection. Bipolar silver electrodes implanted on the XIlth nerve were used for recording whole XIlth nerve activity ipsilaterally and contralaterally to the injection site. Integrated burst activity was generated from the raw electroneurographic recordings. Analysis of burst activity in neurograms was performed on the integrated Xllth nerve signal. G, Time course of the ratio between the area of the synchronic bursts recorded in the injected versus the contralateral (noninjected) sides measured on the integrated XIlth nerve discharge before and after central microinjections of the indicated drugs. Taking as reference the integrated activity from the noninjected control side, $\mathrm{H}-1152$ induced a decrease in the activity recorded ipsilaterally to the injected side, whereas ML-7 induced an increase. Horizontal black and gray lines indicate the timing of injections. $\boldsymbol{H}$, Summary of the effects of vehicle (PBS; $n=11$ injections), H-1152 ( $n=3$ injections in 3 rats), Y-27632 ( $n=6$ injections in 4 rats), and ML-7 ( $n=6$ injections in 3 rats) on the burst area ratio between the injected and control sides obtained when maximal effect was observed (expressed as percentage variation from the control side). ${ }^{*} p<0.05$, Mann-Whitney $U$ test.

Endogenous ROCK signaling facilitates baseline inspiratory activity of the HMN pool in adulthood

To evaluate whether endogenous activity of presynaptic ROCK has any physiologically relevant function in modulating the normal motor output from the HN in adulthood, we first analyzed the expression pattern of ROCK protein within the HN of adult rat brainstems. Accordingly, coronal brainstem sections containing FG-back-labeled HMNs were processed for dual immunodetection of the presynaptic marker syn and either ROCK $\alpha$ or ROCK $\beta$ (Fig. $8 A-E$ ). Our data show that immunolabeling for each of the isoforms had a punctuate pattern throughout the HN that partially overlapped the distribution of syn-ir puncta. Quantitative analysis shows that $22.1 \pm 2.4 \%(n=350$ boutons apposed on $14 \mathrm{HMNs})$ and $21.3 \pm 3.0 \%(n=412$ boutons apposed on 19 HMNs) of syn-ir puncta apposed to FG-identified HMNs colocalized with $\operatorname{ROCK} \alpha$ - or ROCK $\beta$-ir puncta, respectively. Therefore, the molecular machinery to support a role of presynaptic ROCK in neurotransmitter release is also present in the adult HN. Given that ROCK $\alpha$ immunohistochemistry in adults was performed using an antibody dilution higher than that used for neonatal tissue, we cannot discard the possibility that this isoform is actually expressed within adult HMN cell bodies.

Most HMNs discharge bursts of action potentials synchronized with the inspiratory phase of respiration that are driven mainly by glutamatergic brainstem afferences with little or no contribution of inhibitory inputs (Peever et al., 2002; GonzálezForero et al., 2004). This rhythmic activity persists during anesthesia or following decerebration, even though most oro-facial motor drive is suppressed under such conditions (GonzálezForero et al., 2004; González-Forero et al., 2007; Montero et al., 2008; Montero et al., 2010). To determine whether endogenous ROCK also facilitates transmitting and shaping motor commands to the HN in adult animals, we tested the effect of unilateral microinjections of ROCK inhibitors into the HN of anesthetized adult rats on the rhythmic bursting pattern of the XIIth nerve. Activity was expressed as the ratio of the integrated area of XIIth nerve bursts between the injected and contralateral (noninjected) sides. This ratio was significantly decreased after microinjection of H-1152 (2 nmol) into the right nucleus, reaching maximal effects $(-10.0 \pm 1.9 \%)$ at $5.2 \pm 0.2 \mathrm{~min}$ from the 


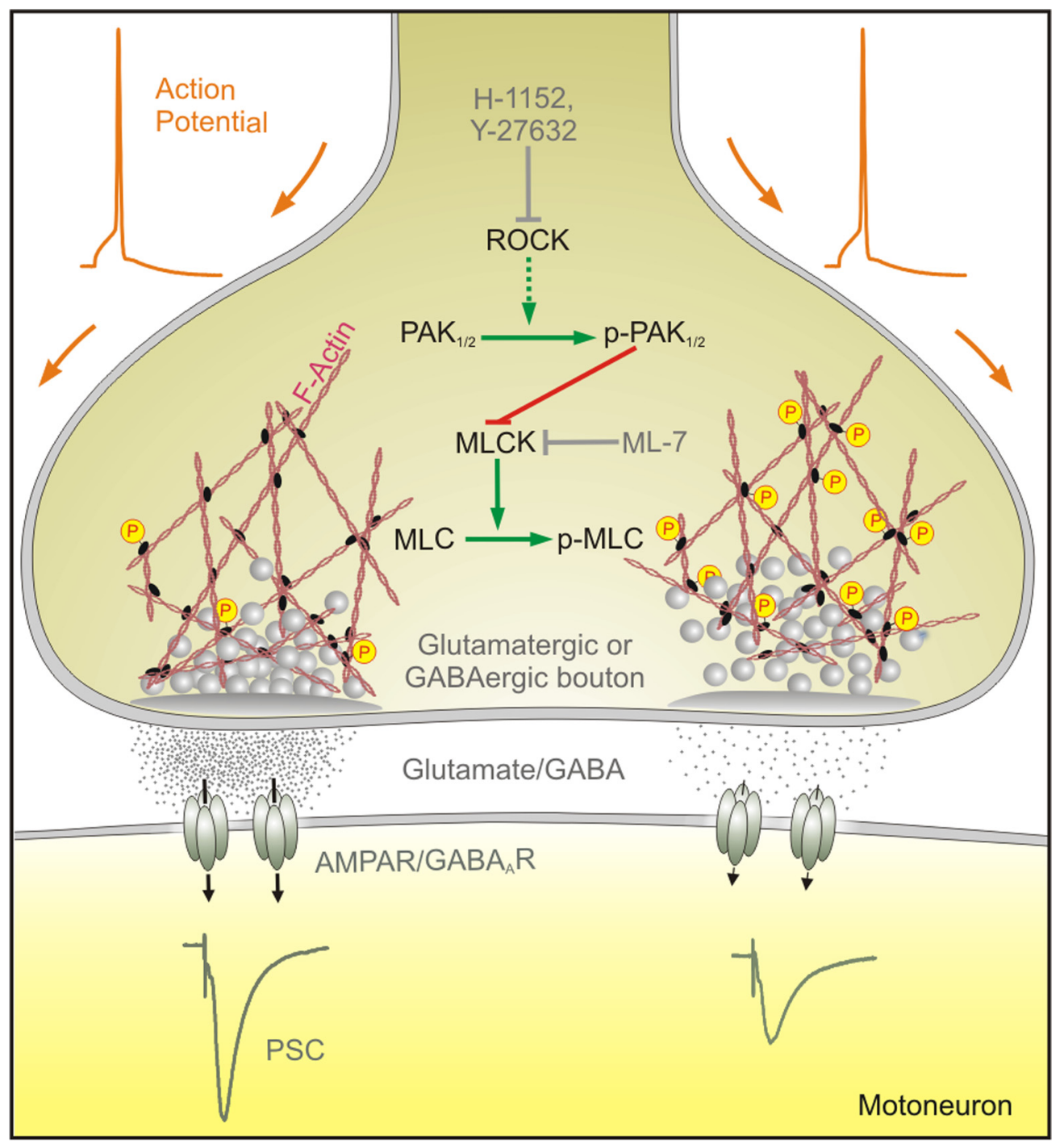

Figure 9. Proposed mechanism by which baseline ROCK activity maintains synaptic strength. Endogenous ROCK signaling, by direct and/or indirect phosphorylation, activates PAK ${ }_{1-3}$, which through an inhibitory phosphorylation directly keeps MLCK in an inhibited state. Subsequently, MLCK inactivation promotes dephosphorylation of MLC and thus slows or arrests contractility of the actomyosin structure. Stabilization of the actomyosin cytoskeleton would be required to maintain the spatial distribution of vesicles within the presynaptic terminal and the size of the RRP of synaptic vesicles. Inhibition of basal ROCK signaling leads to enhanced MLC phosphorylation, contraction of the actomyosin apparatus, and reduction of the size of the RRP of synaptic vesicles, which results in short-term depression of synaptic transmission and in attenuation of AMPAR and GABA $A_{A}$-receptor-mediated postsynaptic currents (PSCs). Pharmacological suppression of endogenous ROCK

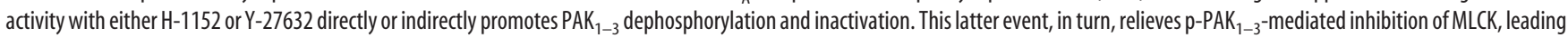
to enhanced MLC phosphorylation. Inhibition of MLCK with ML-7 suppresses the effects of ROCK inhibitors on the phosphorylation level of MLC and on synaptic strength.

beginning of the drug administration (Fig. $8 \mathrm{~F}-H$ ). Intranuclear injection of another ROCK inhibitor, Y-27632 ( $5 \mathrm{nmol})$, induced similar alterations peaking $(-6.5 \pm 0.9 \%)$ at $4.2 \pm 0.8 \mathrm{~min}$ (Fig. $8 \mathrm{H})$. These data demonstrate that endogenous ROCK activity plays an outstanding role in modulating the physiological patterns of HN motor output via facilitation of baseline discharge activity of the HMN pool in adulthood.

Our in vitro experiments on neonatal HMNs provide evidence that ROCK regulates excitatory synaptic strength through MLCK inhibition. Hence, we aimed to investigate a feasible regulatory action of endogenous MLCK on the inspiratory basal activity of the HMN pool in adults. Local microinjection of the MLCK inhibitor ML-7 (2.5 nmol) significantly increased discharge activity recorded in the XIIth nerve ipsilateral to the injected nucleus compared with the noninjected side (Fig. $8 G, H$ ), reaching maximal effects $(+7.7 \pm 0.8 \%)$ at $9.2 \pm 1.0$ min postinjection. These results indicate that endogenous activity of MLCK regulates mo- tor output commands in vivo and also agree with our in vitro findings, suggesting that this kinase exerts an inhibitory influence on glutamate release. Differential action of ML-7 in in vitro and in vivo preparations could be the consequence of different activity states of the afferent drive on HMNs in the two experimental approaches.

\section{Discussion}

We report here that endogenous ROCK signaling is pivotal for the normal performance of motor output commands. ROCK activity maintains synaptic strength by regulating the size of the RRP of synaptic vesicles at afferent synapses driving motoneurons (Fig. 9). The mechanism of action involves imbalance toward the nonphosphorylated form of MLC by indirect inhibition of MLCK, probably by phosphorylation of $\mathrm{PAK}_{1-3}$, which directly inhibits MLCK. By reducing MLC phosphorylation, ROCK might keep the presynaptic actin-myosin contractile apparatus in 
a baseline condition to maintain a determined bulk of the RRP of synaptic vesicles (Fig. 9). Thus, physiological modulators of presynaptic ROCK activity could be feasible triggers for shortterm synaptic plasticity in the nervous system.

Despite the high sequence similarity (83\%) between ROCK $\alpha$ and $\beta$ (Amano et al., 2010), their differential patterns of tissue distribution suggest distinct functions for the two isoforms in vivo. In the brain, $\mathrm{ROCK} \alpha$ is expressed at higher levels than ROCK $\beta$ (Leung et al., 1996; Matsui et al., 1996; Nakagawa et al., 1996). Expression levels of ROCK $\alpha$ gradually increase during postnatal development of the mouse brain (Komagome et al., 2000). In bovine brain, this isoform is profusely expressed in neurons of the cerebral cortex, hippocampus, and cerebellum (Hashimoto et al., 1999). Accordingly, we found that the $\alpha$, but not the $\beta$, isoform is expressed in HMNs from neonatal rats. Additionally, ROCK-ir puncta of both isoforms were observed colocalizing and/or in close proximity to syn- or VGLUT2-ir puncta apposed to HMNs at neonatal stages. This distribution pattern is consistent with a preferential, but not exclusive, postsynaptic action of ROCK $\alpha$ and a presynaptic function of both isoforms. The finding that ROCK-ir punctate partially overlap with syn-ir puncta in the $\mathrm{HN}$ of adult rats further suggests that the presynaptic action of ROCKs is retained at older ages. Interestingly, ROCK-ir puncta colocalize and/or are in close proximity to VGLUT2-ir puncta apposed to adult HMNs (Sunico et al., 2010). Therefore, the molecular substrate for a possible presynaptic role of ROCK in excitatory inputs is also present in the adult HN.

We found that the endogenously expressed ROCK protein retains activity in the $\mathrm{HN}$ from in vitro brainstem slices. However, basal activity of ROCK does not appear to exert any effect on intrinsic membrane properties as suggested by the lack of changes in intrinsic excitability of HMNs below $\sim 62 \%$ ROCK inhibition. This notion is supported by recent data from our lab showing no changes in membrane electrophysiological parameters even after $100 \%$ inhibition of the kinase activity (García-Morales V, González-Forero D, Moreno-López B; unpublished results). These data argue against the idea that endogenous activity of ROCK modulates intrinsic excitability of neonatal HMNs in vitro. This could be explained by lack of regulation of the intrinsic HMN excitability by this isoform, and/or by endogenous activity of motoneuronal ROCK $\alpha$ below the level required to reveal its impact on membrane properties in our experimental conditions. The former possibility is less likely because RhoA/ROCK system modulates the activity of several classes of ion channels (Cachero et al., 1998; Li et al., 2002; Storey et al., 2002; Staruschenko et al., 2004; Iftinca et al., 2007), some of which are present in motoneurons. These findings rule out the possibility that in our experimental preparation the functional alterations induced by ROCK inhibition were contaminated by changes in intrinsic membrane properties of HMNs.

ROCK regulates a wide variety of neuronal functions that are dependent on cytoskeletal control through regulation of the actin-myosin network (Riento and Ridley, 2003; Mueller et al., 2005). The actin-myosin cytoskeletal structure, in turn, regulates distribution and trafficking of synaptic vesicles within presynaptic terminals and neurotransmitter release at synapses (Trifaró and Vitale, 1993; Dillon and Goda, 2005). The idea of a presynaptic locus for a ROCK-mediated modulation of neurotransmission is also supported by the findings that ROCK inhibits N-type $\mathrm{Ca}^{2+}$ channels (Piccoli et al., 2004), which are involved in excitation-secretion coupling at central synapses (Dunlap et al., 1995), and Kv1.2 channels, which are often localized at synaptic compartments (Cachero et al., 1998). In our experimental con- ditions, ROCK inhibition strongly reduced amplitudes of

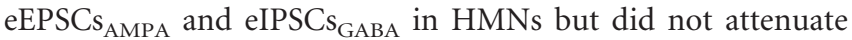
mEPSCs $_{\mathrm{AMPA}}$ or whole-cell responses to glutamate pulses. Strikingly, kinase inhibition elicited a conspicuous depression of action potential- and $\mathrm{Ca}^{2+}$-dependent AMPAR-mediated glutamatergic transmission and increased both the magnitude of facilitation induced by paired pulse or repetitive afferent stimu-

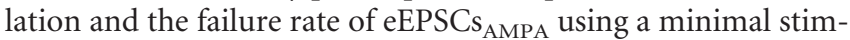
ulation paradigm. Additionally, ROCK inhibition resulted in a reduction in the number of synaptic vesicles docked to the a.z. Altogether, these results strongly indicate that endogenous ROCK signaling maintains normal synaptic transmission through a purely presynaptic mechanism. Ultrastructural correlates for the short-term changes in synaptic strength induced by ROCK inhibition further suggest that they may be ascribed, at least in part, to a reduction in the size of the RRP of synaptic vesicles.

Changes in the actin cytoskeleton are a prerequisite for exocytosis, enabling docking and fusion of secretory vesicles with the plasma membrane (Dillon and Goda, 2005). ROCK directly and/or indirectly, through MLCP inhibition, induces MLC phosphorylation that subsequently stimulates myosin-actin interactions (Mueller et al., 2005). In this context, a physical relationship between ROCK and p-MLC in excitatory synaptic puncta on adult and neonatal HMNs has been recently reported (Sunico et al., 2010). By contrast, in the present study ROCK inhibition induced an unexpected increase in p-MLC levels in the HN from neonatal slices. This agrees with a ROCK-mediated inhibition of MLCK, the main kinase that phosphorylates MLC, rather than with the ROCK-induced MLC phosphorylation reported so far. Coaddition to the bath solution of a specific inhibitor of MLCK fully prevented/reverted all functional and ultrastructural changes induced by ROCK inhibition. These results are consistent with those reported at the calyx of Held synapse, in which MLCK was shown to control the size of the pool of fast releasing vesicles (Srinivasan et al., 2008). The finding that MLCK inhibition per se failed to modify both p-MLC levels and the amplitude of eEPSCs further suggests that basal activity of MLCK in our experimental preparation is low enough so that its effects on the actomyosin apparatus were relegated to the synaptic vesicle pool. This also suggests a MLCK-independent mechanism maintaining baseline MLC phosphorylation levels at the HN. Thus, molecular pathways, differentially modulated and essentially distinct in composition but redundant in their effects, could converge on MLC to stimulate its phosphorylation. It is therefore possible that overstimulation of ROCK does not necessarily result in functional changes exactly opposite to those seen after ROCK inhibition. In fact, the addition to the bath solution of lysophosphatidic acid (LPA), a widely used activator of the RhoA/ROCK signaling pathway, failed to induce potentiation of eEPSCs ${ }_{\text {AMPA }}$ in HMNs or to reduce p-MLC levels in the HN in vitro (González-Forero D, Domínguez G, and Moreno-López B; unpublished results).Together, our functional and anatomical results strongly suggest that endogenous ROCK activity, through inhibition of MLCK, maintains synaptic strength on motoneurons by stabilizing the actomyosin cytoskeleton and the spatial distribution of the RRP of synaptic vesicles in synaptic boutons (Fig. 9).

A link supporting the direct or indirect inhibitory action of ROCK on MLCK is lacking so far. PAK, a downstream effector of the Rho GTPases Rac and Cdc42, is a plausible candidate molecule for mediating ROCK inhibition of MLCK because it catalyzes an inhibitory phosphorylation of MLCK (Li et al., 2010). Phosphorylation of the kinase domain is critical to PAK activa- 
tion (Galan Moya et al., 2009). Accordingly, ROCK inhibition induced a drastic reduction in the phosphorylated forms of $\mathrm{PAK}_{1-3}$ at their kinase domains in the HN. This finding supports a new crosstalk interaction between the molecular pathways downstream of Rac, $\mathrm{Cdc42}$, and RhoA that regulates actinmyosin interactions (Burridge and Wennerberg, 2004). Since the sites of phosphorylation in PAK kinase domains present consensus phosphorylation sequences for ROCK, a direct action of ROCK on PAKs could be possible (Amano et al., 2010). We propose here a new mechanism of action by which endogenous ROCK signaling favors PAK activity by phosphorylation, which directly phosphorylates and inhibits MLCK (Fig. 9).

Administration of specific inhibitors of ROCK or MLCK into the $\mathrm{HN}$ reduced or increased, respectively, the inspiratoryrelated activity recorded in the XIIth nerve. This activity represents the output of the HMN pool, which is driven by AMPAR-mediated excitatory inputs to HMNs (Rekling and Feldman, 1998). These data, together with the finding that excitatory inputs on HMNs express ROCK, strongly indicate that endogenous ROCK activity is required to maintain normal patterns of motor output from the HN in adult animals. Whether the maintenance of baseline ROCK activity in vitro and in vivo is dependent on membrane-derived bioactive lipid mediators, such as LPA and sphingosine 1-phosphate (S1P), requires further investigation. Supporting this hypothesis, it has been recently recognized that LPA and S1P receptors are expressed in the nervous system (Anliker and Chun, 2004; Choi et al., 2010). Likewise, LPA and S1P have been involved in spatial learning (Dash et al., 2004; Kanno et al., 2010), which supports their involvement in synaptic plasticity events.

\section{References}

Amano M, Nakayama M, Kaibuchi K (2010) Rho-kinase/ROCK: A key regulator of the cytoskeleton and cell polarity. Cytoskeleton 67:545-554.

Anliker B, Chun J (2004) Cell surface receptors in lysophospholipid signaling. Semin Cell Dev Biol 15:457-465.

Bähler M, Greengard P (1987) Synapsin I bundles F-actin in a phosphorylation-dependent manner. Nature 326:704-707.

Benarroch EE (2007) Rho GTPases: role in dendrite and axonal growth, mental retardation, and axonal regeneration. Neurology 68:1315-1318.

Burridge K, Wennerberg K (2004) Rho and Rac take center stage. Cell 116:167-179.

Cachero TG, Morielli AD, Peralta EG (1998) The small GTP-binding protein RhoA regulates a delayed rectifier potassium channel. Cell 93:1077-1085.

Carroll RC, Lissin DV, von Zastrow M, Nicoll RA, Malenka RC (1999) Rapid redistribution of glutamate receptors contributes to long-term depression in hippocampal cultures. Nat Neurosci 2:454-460.

Choi JW, Herr DR, Noguchi K, Yung YC, Lee CW, Mutoh T, Lin ME, Teo ST, Park KE, Mosley AN, Chun J (2010) LPA receptors: subtypes and biological actions. Annu Rev Pharmacol Toxicol 50:157-186.

Dash PK, Orsi SA, Moody M, Moore AN (2004) A role for hippocampal Rho-ROCK pathway in long-term spatial memory. Biochem Biophys Res Commun 322:893-898.

Dillon C, Goda Y (2005) The actin cytoskeleton: integrating form and function at the synapse. Annu Rev Neurosci 28:25-55.

Dobrunz LE (2002) Release probability is regulated by the size of the readily releasable vesicle pool at excitatory synapses in hippocampus. Int J Dev Neurosci 20:225-236.

Drenckhahn D, Kaiser HW (1983) Evidence for the concentration of F-actin and myosin in synapses and in the plasmalemmal zone of axons. Eur J Cell Biol 31:235-240.

Dunlap K, Luebke JI, Turner TJ (1995) Exocytotic $\mathrm{Ca}^{2+}$ channels in mammalian central neurons. Trends Neurosci 18:89-98.

Galan Moya EM, Le Guelte A, Gavard J (2009) PAKing up to the endothelium. Cell Signal 21:1727-1737.

González-Forero D, Alvarez FJ (2005) Differential postnatal maturation of
GABAA, glycine receptor, and mixed synaptic currents in Renshaw cells and ventral spinal interneurons. J Neurosci 25:2010-2023.

González-Forero D, Portillo F, Sunico CR, Moreno-López B (2004) Nerve injury reduces responses of hypoglossal motoneurones to baseline and chemoreceptor-modulated inspiratory drive in the adult rat. J Physiol 557:991-1011.

González-Forero D, Portillo F, Gómez L, Montero F, Kasparov S, MorenoLópez B (2007) Inhibition of resting potassium conductances by longterm activation of the NO/cGMP/protein kinase G pathway: A new mechanism regulating neuronal excitability. J Neurosci 27:6302-6312.

Hashimoto R, Nakamura Y, Kosako H, Amano M, Kaibuchi K, Inagaki M, Takeda M (1999) Distribution of Rho-kinase in the bovine brain. Biochem Biophys Res Commun 263:575-579.

Hirokawa N, Sobue K, Kanda K, Harada A, Yorifuji H (1989) The cytoskeletal architecture of the presynaptic terminal and molecular structure of synapsin 1. J Cell Biol 108:111-126.

Huentelman MJ, Stephan DA, Talboom J, Corneveaux JJ, Reiman DM, Gerber JD, Barnes CA, Alexander GE, Reiman EM, Bimonte-Nelson HA (2009) Peripheral delivery of a ROCK inhibitor improves learning and working memory. Behav Neurosci 123:218-223.

Iftinca M, Hamid J, Chen L, Varela D, Tadayonnejad R, Altier C, Turner RW, Zamponi GW (2007) Regulation of T-type calcium channels by Rhoassociated kinase. Nat Neurosci 10:854-860.

Kanno T, Nishizaki T, Proia RL, Kajimoto T, Jahangeer S, Okada T, Nakamura S (2010) Regulation of synaptic strength by sphingosine 1-phosphate in the hippocampus. Neuroscience 171:973-980.

Komagome R, Kimura K, Saito M (2000) Postnatal changes in Rho and Rho-related proteins in the mouse brain. Jpn J Vet Res 47:127-133.

Leung T, Manser E, Tan L, Lim L (1995) A novel serine/threonine kinase binding the Ras-related RhoA GTPase which translocates the kinase to peripheral membranes. J Biol Chem 270:29051-29054.

Leung T, Chen XQ, Manser E, Lim L (1996) The p160 RhoA-binding kinase ROK alpha is a member of a kinase family and is involved in the reorganization of the cytoskeleton. Mol Cell Biol 16:5313-5327.

Li L, Matsuoka I, Suzuki Y, Watanabe Y, Ishibashi T, Yokoyama K, Maruyama Y, Kimura J (2002) Inhibitory effect of fluvastatin on lysophosphatidylcholine-induced nonselective cation current in Guinea pig ventricular myocytes. Mol Pharmacol 62:602-607.

Li T, Fang Y, Yang G, Zhu Y, Xu J, Liu L (2010) The mechanism by which RhoA regulates vascular reactivity after hemorrhagic shock in rats. Am J Physiol Heart Circ Physiol 299:H292-299.

Llinás R, McGuinness TL, Leonard CS, Sugimori M, Greengard P (1985) Intraterminal injection of synapsin I or calcium/calmodulin-dependent protein kinase II alters neurotransmitter release at the squid giant synapse. Proc Natl Acad Sci U S A 82:3035-3039.

Luo L (2002) Actin cytoskeleton regulation in neuronal morphogenesis and structural plasticity. Annu Rev Cell Dev Biol 18:601-635.

Matsui T, Amano M, Yamamoto T, Chihara K, Nakafuku M, Ito M, Nakano T, Okawa K, Iwamatsu A, Kaibuchi K (1996) Rho-associated kinase, a novel serine/threonine kinase, as a putative target for small GTP binding protein Rho. EMBO J 15:2208-2216.

Millar AG, Bradacs H, Charlton MP, Atwood HL (2002) Inverse relationship between release probability and readily releasable vesicles in depressing and facilitating synapses. J Neurosci 22:9661-9667.

Montero F, Portillo F, González-Forero D, Moreno-López B (2008) The nitric oxide/cyclic guanosine monophosphate pathway modulates the inspiratory-related activity of hypoglossal motoneurons in the adult rat. Eur J Neurosci 28:107-116.

Montero F, Sunico CR, Liu B, Paton JF, Kasparov S, Moreno-López B (2010) Transgenic neuronal nitric oxide synthase expression induces axotomylike changes in adult motoneurons. J Physiol 588:3425-3443.

Moreno-López B, Sunico CR, González-Forero D (2011) NO orchestrates the loss of synaptic boutons from adult "sick" motoneurons: modeling a molecular mechanism. Mol Neurobiol 43:41-66.

Mueller BK, Mack H, Teusch N (2005) Rho kinase, a promising drug target for neurological disorders. Nat Rev Drug Discov 4:387-398.

Nakagawa O, Fujisawa K, Ishizaki T, Saito Y, Nakao K, Narumiya S (1996) ROCK-I and ROCK-II, two isoforms of Rho-associated coiled-coil forming protein serine/threonine kinase in mice. FEBS Lett 392:189-193.

Ota KT, Monsey MS, Wu MS, Schafe GE (2010) Synaptic plasticity and NO-cGMP-PKG signaling regulate pre- and postsynaptic alterations at 
rat lateral amygdala synapses following fear conditioning. PLoS One 5:e11236.

Peever JH, Shen L, Duffin J (2002) Respiratory pre-motor control of hypoglossal motoneurons in the rat. Neuroscience 110:711-722.

Phillips GR, Huang JK, Wang Y, Tanaka H, Shapiro L, Zhang W, Shan WS, Arndt K, Frank M, Gordon RE, Gawinowicz MA, Zhao Y, Colman DR (2001) The presynaptic particle web: ultrastructure, composition, dissolution, and reconstitution. Neuron 32:63-77.

Piccoli G, Rutishauser U, Brusés JL (2004) N-cadherin juxtamembrane domain modulates voltage-gated $\mathrm{Ca}^{2+}$ current via RhoA GTPase and Rhoassociated kinase. J Neurosci 24:10918-10923.

Rekling JC, Feldman JL (1998) PreBotzinger complex and pacemaker neurons: hypothesized site and kernel for respiratory rhythm generation. Annu Rev Physiol 60:385-405.

Riento K, Ridley AJ (2003) Rocks: multifunctional kinases in cell behaviour. Nat Rev Mol Cell Biol 4:446-456.

Sakaba T, Neher E (2003) Involvement of actin polymerization in vesicle recruitment at the calyx of Held synapse. J Neurosci 23:837-846.

Schiebler W, Jahn R, Doucet JP, Rothlein J, Greengard P (1986) Characterization of synapsin I binding to small synaptic vesicles. J Biol Chem 261:8383-8390.

Srinivasan G, Kim JH, von Gersdorff H (2008) The pool of fast releasing vesicles is augmented by myosin light chain kinase inhibition at the calyx of Held synapse. J Neurophysiol 99:1810-1824.

Staruschenko A, Nichols A, Medina JL, Camacho P, Zheleznova NN, Stock- and JD (2004) Rho small GTPases activate the epithelial $\mathrm{Na}(+)$ channel. J Biol Chem 279:49989-49994.

Storey NM, O'Bryan JP, Armstrong DL (2002) Rac and Rho mediate opposing hormonal regulation of the ether-a-go-go-related potassium channel. Curr Biol 12:27-33.

Sunico CR, González-Forero D, Domínguez G, García-Verdugo JM, Moreno-López B (2010) Nitric oxide induces pathological synapse loss by a protein kinase G-, Rho kinase-dependent mechanism preceded by myosin light chain phosphorylation. J Neurosci 30:973-984.

Sunico CR, Domínguez G, García-Verdugo JM, Osta R, Montero F, MorenoLópez B (2011) Reduction in the motoneuron inhibitory/excitatory synaptic ratio in an early-symptomatic mouse model of amyotrophic lateral sclerosis. Brain Pathol 21:1-15.

Trifaró JM, Vitale ML (1993) Cytoskeleton dynamics during neurotransmitter release. Trends Neurosci 16:466-472.

Turrigiano GG, Leslie KR, Desai NS, Rutherford LC, Nelson SB (1998) Activity-dependent scaling of quantal amplitude in neocortical neurons. Nature 391:892-896.

Wang HG, Lu FM, Jin I, Udo H, Kandel ER, de Vente J, Walter U, Lohmann SM, Hawkins RD, Antonova I (2005) Presynaptic and postsynaptic roles of NO, cGK, and RhoA in long-lasting potentiation and aggregation of synaptic proteins. Neuron 45:389-403.

Zucker RS (1989) Short-term synaptic plasticity. Annu Rev Neurosci 12: $13-31$. 